\title{
Operation Mechanism of Organic Electrochemical Transistors as Redox Chemical Transducers
}

Siew Ting Melissa Tan ${ }^{1}$, Scott Keene ${ }^{2}$, Alexander Giovannitti ${ }^{1}$, Armantas Melianas ${ }^{1}$, Maximilian Moser $^{3}$, Iain McCulloch ${ }^{3,4}$, Alberto Salleo ${ }^{1 *}$

${ }^{1}$ Department of Materials Science and Engineering, Stanford University, California 94305, USA

${ }^{2}$ Electrical Engineering Division, Department of Engineering, University of Cambridge, Cambridge, United Kingdom

${ }^{3}$ Department of Chemistry, Chemistry Research Laboratory, University of Oxford, Oxford, OX1 3TA, UK

${ }^{4}$ Physical Science and Engineering Division, King Abdullah University of Science and Technology, Thuwal 23955-6900, Saudi Arabia

*Corresponding author email: asalleo@stanford.edu

\section{ToC}



Designing Organic Electrochemical Transistors to transduce and amplify chemical redox reactions while maintaing stable and reliable performance requires: 1) separating the redox reaction from the OECT 2) operating the OECT potentiometrically 3) utilizing a non-polarizable gate electrode 4) selecting an appropriate channel material to achieve high transconductance. 


\begin{abstract}
The ability to control the charge density of organic mixed ionic electronic conductors (OMIECs) via reactions with redox-active analytes has enabled applications as electrochemical redox sensors. Their charge density-dependent conductivity can additionally be tuned via charge injection from electrodes, for instance in organic electrochemical transistors (OECTs), where volumetric charging of the OMIEC channel enables excellent transconductance and amplification of low potentials. Recent efforts have combined the chemical detection with the transistor function of OECTs to achieve compact electrochemical sensors. However, these sensors often fall short of the expected amplification performance of OECTs. Here, we investigate the operation mechanism of various OECT architectures to deduce the design principles required to achieve reliable chemical detection and signal amplification. By utilizing a non-polarizable gate electrode and conducting the chemical reaction in a compartment separate from the OECT, the recently developed Reaction Cell OECT achieves reliable modulation of the OECT channel's charge density. This work demonstrates that systematic and rational design of OECT chemical sensors requires understanding the electrochemical processes that result in changes in the potential (charge density) of the channel, the underlying phenomenon behind amplification.
\end{abstract}

\title{
Introduction
}

Organic mixed ionic electronic conductors (OMIECs), commonly conjugated polymers, are promising materials for biosensors because they are well-suited for detecting chemical analytes, especially in aqueous media, due to their redox-activity as well as ionic and electronic conductivity. ${ }^{[1]}$ Charge transfer processes, via faradaic reactions with chemically reactive redox analytes or capacitive charge injection from conductive electrodes, change the charge density on their backbone and consequently the electrochemical potential of electrons. As with any semiconductor, charge density (related to the position of the Fermi level in the OMIEC) modulates electronic conductivity, which can be measured by applying a probing bias and measuring the ensuing current. Charge density also affects the OMIECs' electronic charge mobility and volumetric capacitance, giving rise to second-order effects on the materials' conductivity. Furthermore, side chain engineering enables the intercalation of charge compensating ions to permeate the bulk, facilitating operation in aqueous electrolytes and volumetric charging throughout the material. ${ }^{[2-7]}$ These multifunctional properties of OMIECs have enabled their applications in a variety of electrochemical devices such as sensor electrodes in 3-electrode electrochemical cells to detect redox-active analytes. ${ }^{[8]}$ Additionally, control of electronic conductivity with charge density (or, equivalently, potential) has enabled their usage as the channel material in organic electrochemical transistors (OECTs). ${ }^{[9-15]}$

Operation of an OECT in the traditional sense is potentiometric - changes in potential difference between the gate electrode and the channel modulate the latter's charge density and hence conductivity. Conventionally, the source electrode is grounded and the gate potential is measured with respect to the source $\left(V_{\mathrm{GS}}\right)$. The change in conductivity can be probed by application of a drain bias with respect to the source $\left(V_{\mathrm{DS}}\right)$ across the channel and measuring changes in the drain

current $\left(I_{\mathrm{D}}\right)$. Transconductance $\left(g_{m}=\frac{\Delta I_{D}}{\Delta V_{G S}}\right)$ is the figure of merit that captures the sensitivity of 
channel conductivity in response to a change in its potential or, equivalently and perhaps more intuitively, its charge density. Due to the ability of the OMIEC channel to charge volumetrically, OECTs display exceptionally large transconductances, allowing deep modulations in channel conductivity to arise from small $V_{\mathrm{GS}}$ modulations, thus amplifying minute potential perturbations. ${ }^{[15-17]}$

Recognizing that OMIECs can function as redox-active electrodes and as the channel material of OECT amplifiers, it is convenient to combine both functionalities within the same device to yield a compact amplifying sensor. Indeed, there have been multiple reports utilizing OECTs as chemical redox sensors, where the analyte is introduced directly into the OECT electrolyte while an external $V_{\mathrm{GS}}$ is applied. ${ }^{[18-21]}$ However, this approach, as we ${ }^{[22]}$ and Macchia et al. ${ }^{[23]}$ have pointed out, merits further analysis. Firstly, the amplification of these reactions by the OECT is unclear. Indeed, all-in-one operation of an OECT sensor with a non-polarizable $\mathrm{Ag} / \mathrm{AgCl}$ gate showed sub-unity current gain. ${ }^{[22]}$ Furthermore, conducting redox reactions within the electrolyte of the OECT in the presence of asymmetric electric fields (from both $V_{\mathrm{GS}}$ and $V_{\mathrm{DS}}$ ) results in complex and site-specific side reactions on both the channel and gate as these parasitic redox reactions may be triggered only at specific voltages. These unknown parasitic reaction currents are superimposed onto the desired sensing current. Thus, while using the OECT as a sensor does provide a response to the analyte concentration, a deeper understanding of the device physics is needed in order to understand whether this architecture is truly advantageous.

To fully leverage the excellent transconductance of the OECT and avoid parasitic side reactions, we proposed a different device architecture, a Reaction Cell OECT (RC-OECT), ${ }^{[22]}$ that first transduces the redox reaction into a potential change that subsequently gates the OECT. By conducting the redox reaction on a separate 2-electrode cell from the OECT, we avoid any side reactions within the OECT. This architecture enables the OECT to truly amplify the redox reaction in the RC, resulting in far larger modulations in OECT channel conductivity than the all-in-one approach.

We emphasize that our analysis pertains to sensing via faradaic redox chemical reactions, where sustained electron transfer occurs between species in the electrolyte and the device electrodes, and is hence distinct from capacitive potentiometric devices e.g. Ion-Selective FETs. Faradaic reactions in the $\mathrm{RC}$ enable the $\mathrm{RC}$ (and its corresponding gate circuit) self-powered, distinguishing it from other two-compartment device architectures that operate capacitively e.g. Floating Gate FETs. ${ }^{[24,25]}$

The RC-OECT may be one of many potential approaches to designing redox chemical sensors that fully take advantage of the transconductance of the OECT. To enable further development of highgain chemical transducers based on OECTs, it is important to fundamentally understand the device physics of all OECT-based sensing approaches. To do so, we investigate the electrochemical mechanisms that enable changes in the potential (charge density) of the OECT channel, which is the underlying phenomena behind the OECT's amplification abilities.

Similar to our initial RC-OECT study, $\mathrm{H}_{2} \mathrm{O}_{2}$, which is reduced to $\mathrm{H}_{2} \mathrm{O}$ via the Hydrogen Peroxide Reduction Reaction (HPRR, $\mathrm{H}_{2} \mathrm{O}_{2}+2 \mathrm{H}^{+}+2 e^{-} \rightarrow 2 \mathrm{H}_{2} \mathrm{O}$ ), is utilized as the model redox-active 
species due to its ubiquity in electrochemical sensors. We similarly utilize a homo-3,3'dialkoxybithiophene polymer, p(g3T2), ${ }^{[26]}$ a p-type conjugated polymer with glycolated sidechains, as the model sensing OMIEC in the OECT channel and the RC anode since we found it to be readily oxidized by $\mathrm{H}_{2} \mathrm{O}_{2}$. This study delves into greater details of the electrochemical processes occurring at various electrodes while combining the operational understanding of transistors. We chose a model "analyte" and a model OMIEC, nevertheless our conclusions are general. We envision that this study will enable the systematic optimization of RC-OECT devices as well as spur further designs of potentiometric OECT-based chemical sensors.

\section{Results}
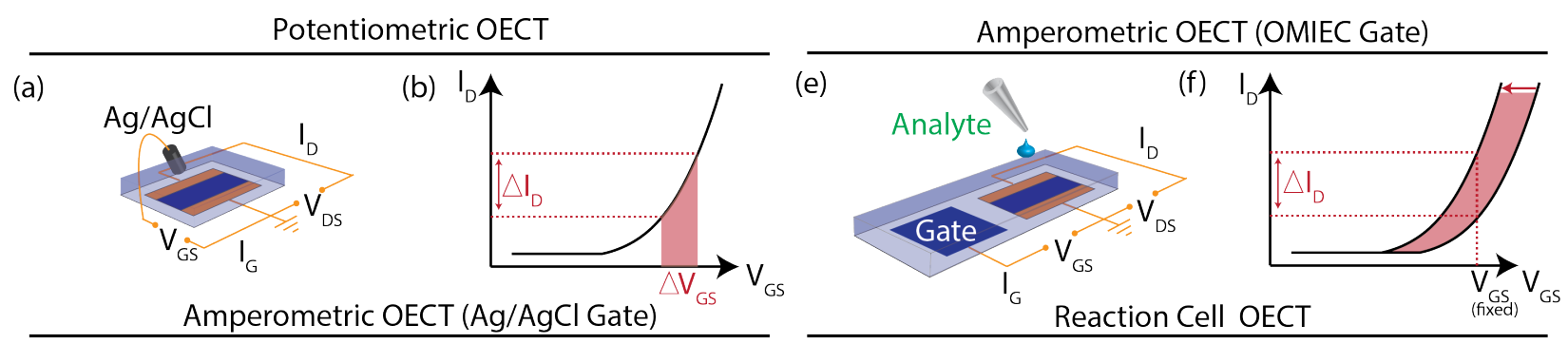

(c)

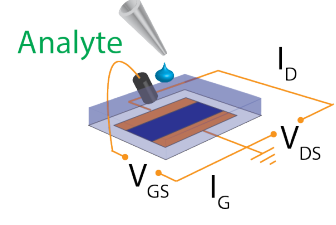

(d)

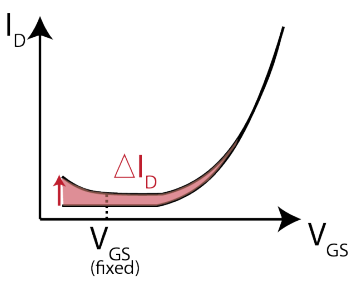

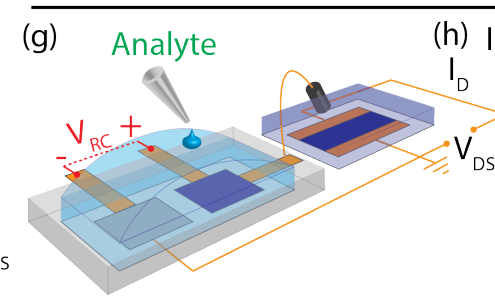

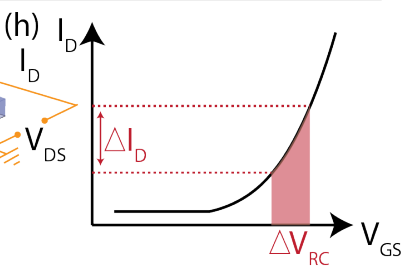

Figure 1. Schematics of various electrochemical device architectures for redox analyte detection. (a) Standard Organic Electrochemical Transistor with OMIEC as the channel between two source and drain contacts as well as an $\mathrm{Ag} / \mathrm{AgCl}$ pellet pseudo-reference electrode as gate immersed in aqueous electrolyte. (b) Schematic of transfer curve of an OECT where a change in drain current $\left(\Delta I_{\mathrm{D}}\right)$ is measured upon applying a change in gate voltage $\left(\Delta V_{\mathrm{GS}}\right)$. (c) Amperometric OECT with an $\mathrm{Ag} / \mathrm{AgCl}$ pellet as gate electrode as well as addition of redox analyte to OECT electrolyte while applying a fixed $V_{\mathrm{GS}}$. (d) Transfer curve showing an increase in faradaic currents upon addition of redox analyte to OECT electrolyte (e) Amperometric OECT with an OMIEC as gate electrode as well as addition of redox analyte to OECT electrolyte while applying a fixed $V_{\mathrm{GS}}$. (f) Schematic of shift in threshold voltage and change in transfer curve of A-OECT (OMIEC gate) after addition of redox analyte to OECT electrolyte. At the fixed $V_{\mathrm{GS}}$, a $\Delta I_{\mathrm{D}}$ arises from the shift in threshold voltage. (g) Reaction Cell OECT where the redox analyte is added to an electrochemical cell separate from the OECT, resulting in a potential change $\left(V_{\mathrm{RC}}\right)$ that is utilized to gate the OECT. (h) Transfer curve of OECT where $\Delta I_{\mathrm{D}}$ is a result of the change in $V_{\mathrm{GS}}=V_{\mathrm{RC}}$.

Traditionally, the most reliable sensing approach of faradaic redox reactions is operation in a 3electrode electrochemical cell, where the OMIEC working electrode is deposited on a current collecting substrate and immersed in an electrolyte. The OMIEC's potential is measured against a standard reference electrode (e.g. $\mathrm{Ag} / \mathrm{AgCl}$ in saturated $\mathrm{KCl}$ for aqueous electrolytes). While utilizing the true reference electrode results in reliable measurements, it is difficult to miniaturize 
and integrate into devices that are micro or nanofabricated. Hence, other electrochemical device architectures have been developed to enable the utilization of a "reference electrode" that is compatible with these fabrication techniques.

The OECT is in fact one such alternative electrochemical device. Most commonly, an $\mathrm{Ag} / \mathrm{AgCl}$ pellet is utilized as pseudo-reference electrode and simultaneously gate electrode for the OECT (Figure 1 (a)). To integrate the gate electrode with microfabricated devices, the $\mathrm{Ag} / \mathrm{AgCl}$ pellet can be miniaturized by depositing $\mathrm{Ag}$ and partially reacting it with $\mathrm{HCl}$ to form $\mathrm{Ag} / \mathrm{AgCl}$. The pellet is not a true reference electrode because it is immersed in an OECT electrolyte where the $\mathrm{Cl}^{-}$concentration is different from that of the saturated electrode and in principle can vary, if for instance $\mathrm{Cl}^{-}$is absorbed in the p-type OMIEC to compensate for hole injection. Typically, one relies on maintaining a high $\mathrm{Cl}^{-}$concentration in the electrolyte to consider it approximately constant. Furthermore, the OECT is inherently a 2-electrode electrochemical cell comprising of the channel (working electrode) and the gate electrode (pseudo-reference), with the absence of a current-collecting counter electrode. Hence, gate currents flow through the gate electrode to compensate for the change in charge on the channel. In typical OECT operation, the device finds itself in the limit of small current flows $(\sim \mathrm{nA})$. Under these conditions the 3-phase equilibrium at the $\mathrm{Ag} / \mathrm{AgCl}$ gate electrode is largely unperturbed, ensuring that the gate potential is fixed, and by virtue of applying a $V_{\mathrm{GS}}$, the OMIEC channel potential can be reliably determined as well. Figure 1 (b) is a schematic of a typical transfer curve of such an OECT where the change in $V_{\mathrm{GS}}$ (red rectangle) modulates the drain current $(\Delta I \mathrm{D})$. Its characteristics can be approximately modelled by the following equation: ${ }^{[13]}$

$$
I_{D}=-\mu C_{c}^{*} \frac{W d}{2 L} \frac{\left[V_{G}-V_{T}\right]^{2}}{V_{T}}, V_{D}<V_{G}-V_{T}
$$

Where $\mu$ and $C_{c}^{*}$ are the charge carrier mobility and volumetric capacitance of the OMIEC channel material respectively, $\frac{W d}{L}$ summarizes the channel geometry, where $W, d$, and $L$ are the channel width, thickness, and length, respectively. $V_{\mathrm{T}}$ is the threshold voltage of the OECT, related to the oxidation or reduction onset of the channel as well as the gate electrode's work function.

Instead of a pseudo-reference electrode, the gate electrode can be of the same material as the channel for ease in depositing and patterning the same material on both the gate and channel in a single deposition step. ${ }^{[11,20]}$ However, the lack of a reference electrode poses challenges in determining the channel's potential (and therefore charge density). The transistor's transfer characteristics - the value of $V_{\mathrm{T}}$ in particular - are strongly dependent on the work function of the gate electrode. ${ }^{[27]} \mathrm{The} \mathrm{Ag} / \mathrm{AgCl}$ gate electrode's work function is reasonably constant, the work function of an OMIEC gate electrode however may vary depending on its processing history and redox reactions with other species present in the electrolyte (e.g. molecular oxygen). ${ }^{[28]}$ Applying $V_{\mathrm{GS}}$ only determines the potential difference between the gate and channel but does not control the potentials of either electrode (hence the position of the Fermi level) with respect to a reference. This leads to many challenges in operating an OECT with OMIEC gate electrodes.

In this study, we compare two commonly seen all-in-one designs in literature and the RC-OECT ${ }^{[22]}$ as redox chemical sensors. The first is an amperometric OECT with $\mathrm{Ag} / \mathrm{AgCl}$ gate electrode 
(Figure 1 (c)). Due to the fixed potential of the gate and the fixed potential difference between gate and source $\left(V_{\mathrm{GS}}\right)$, the potential on the channel is pinned, which prevents any permanent charge accumulation in the channel that otherwise would lead to amplification: every charge introduced in the channel must be swept out, otherwise its potential would change. Hence, changes to drain current equal the total reaction current due to current continuity within the device. It is noteworthy that these currents are potential-dependent (Figure 1 (d)). Alternatively, the gate electrode can be an OMIEC (Figure 1 (e)). As the gate electrode's work function (potential) is not fixed, the channel potential is no longer pinned and the energy of the electrons that could react in the channel is not controlled. Hence, charge transfer reactions on either the gate or channel lead to changes in their potentials, resulting in a shift in the OECT's threshold voltage, $V_{\mathrm{T}}$ as the position of the Fermi level in the semiconductor is no longer fixed (Figure 1 (f)). Lastly, the reaction can instead be separated on a reaction cell where the change in potential across the 2-electrode cell, akin to a $V_{\mathrm{GS}}$, is used to gate the OECT using a non-polarizable gate electrode (Figure 1 (g)). The OECT is operated in a similar manner as Figure 1 (a and b) but the origin of $V_{\mathrm{GS}}$ is now $V_{\mathrm{RC}}$, the reaction cell voltage (Figure $1 \mathbf{( h )}$ ). The following sections discuss the physical origins for the transfer curve behaviors and the respective merits of the various OECT architectures in Figures 1 (d, $f$ and h). 


\section{Effect of gate material on OECT operation}

(a)

(b)
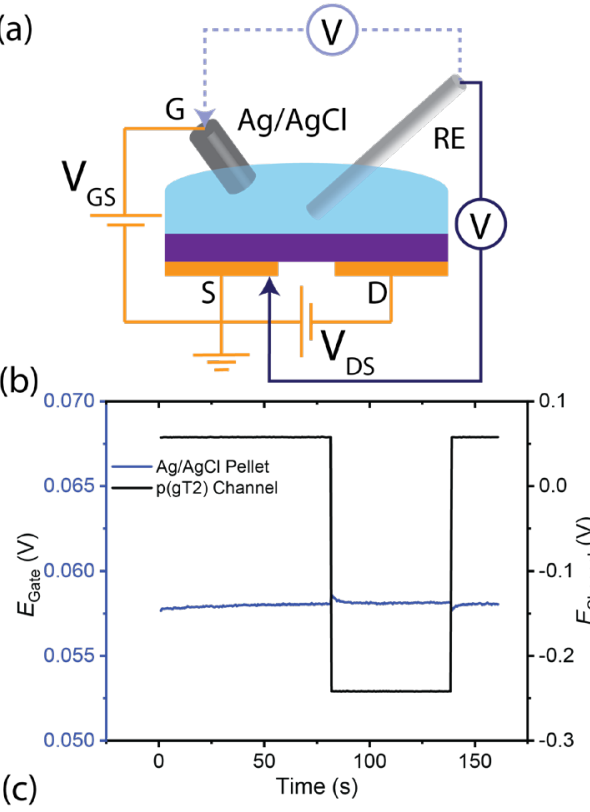

(c)

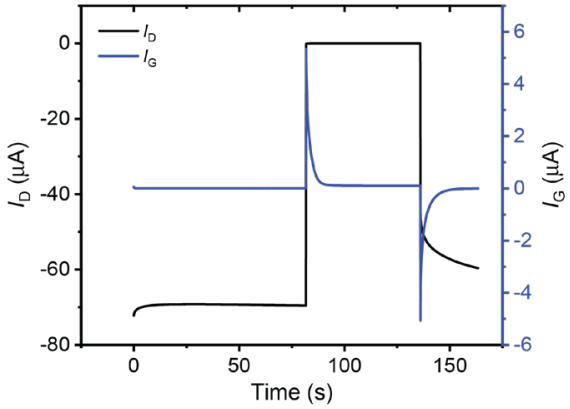

(d)

(e)
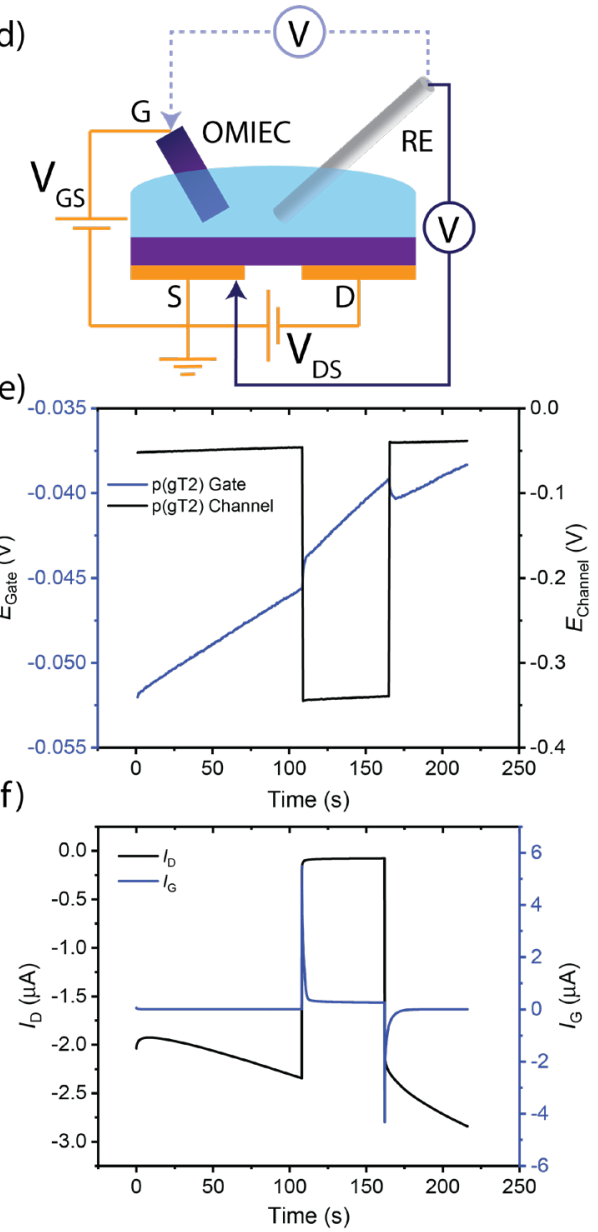

Figure 2. Potential and current stabilities of OECTs using Ag/AgCl vs OMIEC gate electrodes upon pulsing $\boldsymbol{V}_{\mathbf{G}}$ from 0 to $+\mathbf{0 . 3} \mathrm{V}$. (a) Schematic of OECT with $\mathrm{Ag} / \mathrm{AgCl}$ gate while conducting potential measurements of the $\mathrm{Ag} / \mathrm{AgCl}$ gate and source of the channel. (b) Plot of gate (blue) and source (black) potentials in an OECT utilizing $\mathrm{Ag} / \mathrm{AgCl}$ pellet as the gate electrode. (c) Plot of gate and drain current in an OECT utilizing $\mathrm{Ag} / \mathrm{AgCl}$ pellet as the gate electrode. (d) Schematic of OECT with $\mathrm{Ag} / \mathrm{AgCl}$ gate while conducting potential measurements of the $\mathrm{Ag} / \mathrm{AgCl}$ gate and source of the channel. (e) Plot of gate (blue) and source (black) potentials in an OECT utilizing OMIEC as the gate electrode. (f) Plot of gate and drain current in an OECT utilizing OMIEC as the gate electrode.

We first investigate the operation mechanism of an OECT in the absence of any redox chemicals using different gate electrodes: a pseudo-reference electrode $(\mathrm{Ag} / \mathrm{AgCl})$ vs. an OMIEC (p(g3T2)). Utilizing $\mathrm{Ag} / \mathrm{AgCl}$ as the gate electrode results in more stable OECT operation compared to an OMIEC because of the $\mathrm{Ag}(\mathrm{s}) / \mathrm{AgCl}(\mathrm{s}) / \mathrm{Cl}^{-}(\mathrm{aq}) 3$-phase equilibrium. Hence, the potential of the $\mathrm{Ag} / \mathrm{AgCl}$ gate electrode remains constant during regular OECT operation. By applying a fixed $V_{\mathrm{GS}}$, the channel potential and thus its charge density and conductivity can be measured reproducibly. 
This reproducible and deterministic OECT operation is readily verified experimentally (Figure 2 (b and c)).

In contrast, utilizing an OMIEC gate electrode (Figure 2 (d)) of less well-defined potential may lead to challenges in fixing the potential of the channel. The potential of the OMIEC electrode can vary depending on the material's energy levels (which additionally determines its susceptibility to side reactions with ambient oxidants such as oxygen) as well as its processing history and microstructure. The observed drift in potential on the gate over time is likely due to possible side reactions with molecular oxygen dissolved in the electrolyte, ${ }^{[28]}$ resulting in the steady oxidation of the OMIEC gate. Hence, applying constant $V_{\mathrm{GS}}=0 \mathrm{~V}$, the drift in gate potential is reflected in a concomitant drift in channel (source) potential (Figure 2 (e)). Due to the dependence of carrier mobility on charge density and hence potential, the drift in channel potential results in drain current drifts (Figure 2 (f)). We note that this instability may arise for any gate electrode material that is susceptible to parasitic redox side reactions, or any other electronic, chemical, or microstructural changes that may occur during device operation.

In summary, a gate electrode with a stable redox couple ensures that by applying a known $V_{\mathrm{GS}}$, the position of the Fermi level (i.e. the electrochemical potential of the electrons) in the channel can be reproducibly and definitively fixed. 
Effect of faradaic chemical reactions in electrochemical cells

(a) Amperometric OECT (Ag/AgCl Gate)

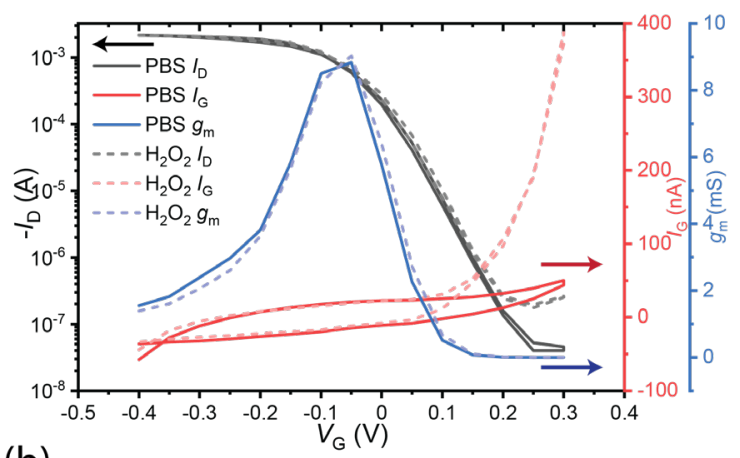

(b)

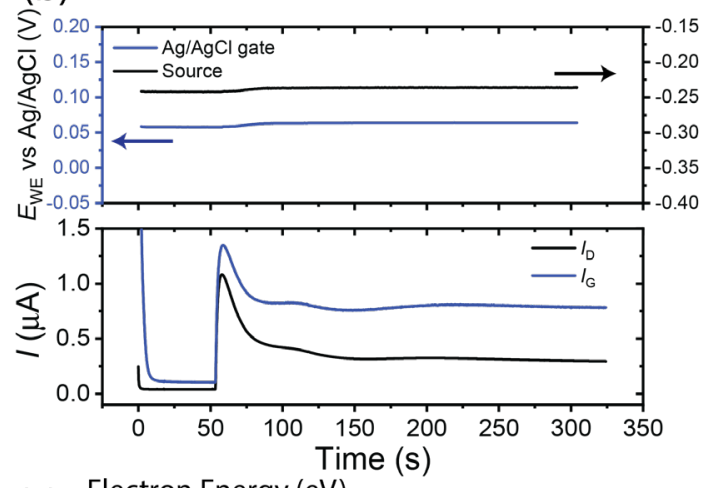

(c)

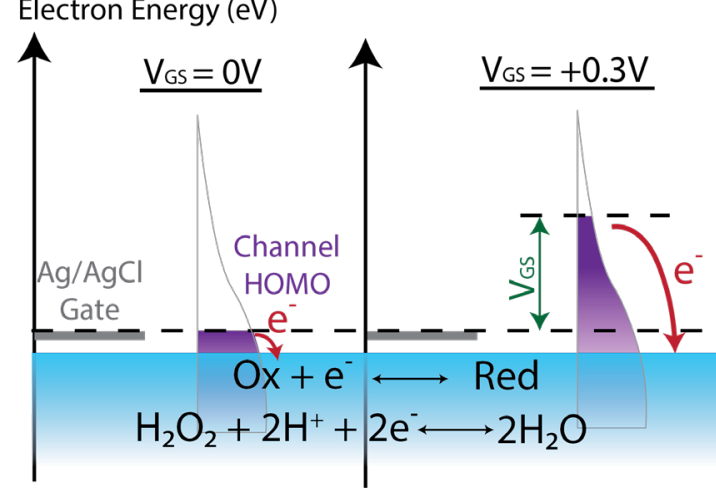

(d) Amperometric OECT (OMIEC Gate)
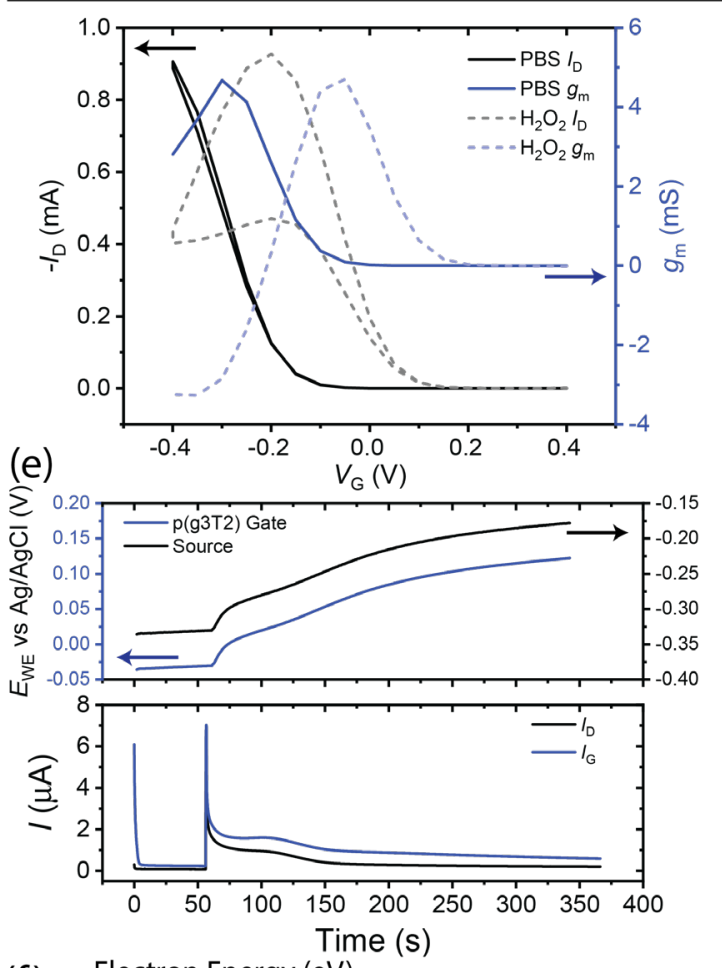

(f)

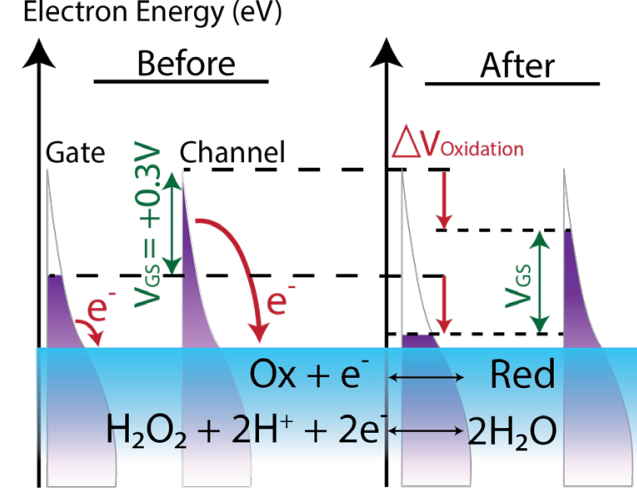

Figure 3. Operation mechanism of Amperometric all-in-one OECTs comparing gate electrodes $\mathrm{Ag} / \mathrm{AgCl}$ and OMIEC. (a-c) $\mathrm{Ag} / \mathrm{AgCl}$ gate. (d-f) OMIEC gate (a) Transfer curve (black), gate currents (red) and transconductance (blue) of the amperometric OECT in its initial state (solid traces) and after addition of the redox analyte to the electrolyte (dashed). (b) Top: Plot of $\mathrm{Ag} / \mathrm{AgCl}$ gate and source potentials vs. a saturated $\mathrm{Ag} / \mathrm{AgCl}$ reference electrode with $V_{\mathrm{GS}}=+0.3$ V. Redox analyte is added to electrolyte at 60 s. Bottom: Corresponding plot of gate and drain currents. (c) Schematic of electron energy levels (defined as negative potentials, - E) in the $\mathrm{Ag} / \mathrm{AgCl}$ pellet gate electrode (grey) and the valence band, Highest Occupied Molecular Orbital (HOMO), of the OMIEC channel (purple) at $V_{\mathrm{GS}}=0 \mathrm{~V}$ (left) and $V_{\mathrm{GS}}=+0.3 \mathrm{~V}$ (right) as well as the energy level of electrons in the redox analyte (blue). Red arrows indicate the flow of electrons from the channel to the analyte in solution. (d) Transfer curve (black) and transconductance (blue) 
of the amperometric OECT with OMIEC gate in its initial state (solid traces) and after addition of the redox analyte to the electrolyte (dashed). (e) Top: Plot of OMIEC gate and source potentials vs. a saturated $\mathrm{Ag} / \mathrm{AgCl}$ reference electrode with $V_{\mathrm{GS}}=+0.3 \mathrm{~V}$. Redox analyte is added to electrolyte at $60 \mathrm{~s}$. Bottom: Corresponding plot of gate and drain currents. (f) Schematic of electron energy levels in the valence bands of the OMIEC gate electrode (left) and OMIEC channel (right) at $V_{\mathrm{GS}}=+0.3 \mathrm{~V}$ as well as the energy level of electrons in the redox analyte. Before: Upon addition of redox analyte to the electrolyte, red arrows indicate flow of electrons from OMIEC electrodes to redox analyte. After: The resulting oxidation of both the gate and channel results in the shift in potentials of both gate and channel by $\Delta \mathrm{V}_{\text {Oxidation. }}$

While utilizing a pseudo-reference gate electrode results in stable and reproducible performance, this all-in-one OECT architecture does not amplify chemical electron transfer reactions. ${ }^{[22]}$ After addition of $\mathrm{H}_{2} \mathrm{O}_{2}$ to the electrolyte, large gate currents occur at positive gate voltages $V_{\mathrm{GS}}>+0.1$ $\mathrm{V}$ in Figure 3 (a), corresponding to the accelerated rate of HPRR at negative channel potentials (SI Figure 1). However, $I_{\mathrm{D}}(\sim 0.5 \mu \mathrm{A})$ is less than $I_{\mathrm{G}}(\sim 1 \mu \mathrm{A})$ (Figure 3 (b)) due to current continuity illustrated in SI Figure 2 (b). The sum of currents across the whole channel (from source to drain) must equal the gate current. Hence, the change in drain current is a fraction of the total gate current, resulting in sub-unity current gains.

Addition of $\mathrm{H}_{2} \mathrm{O}_{2}$ also results in small increase in potential $\sim 5 \mathrm{mV}$ on both the gate and source (Figure 3 (b)). Ideally, the $\mathrm{Ag} / \mathrm{AgCl}$ gate should not experience a potential shift due to its infinite capacitance. However, the large reaction current flowing through the gate (due to absence of a current collecting counter electrode) may have resulted in significant oxidation of Ag, changing the electrochemical potential of the gate electrode. The pinning of the channel potential to the gate potential by applying a fixed $V_{\mathrm{GS}}$ results in the former mirroring the latter. This potential shift is small here, therefore its effect may not be very deleterious. Nevertheless, the shift will depend on the faradaic current, e.g. the analyte concentration, and can therefore in principle be much larger leading to the same problems outlined in the description of the OECT operated with an OMIEC gate electrode in the next section.

The magnitude of reaction currents may be controlled by the applied $V_{\mathrm{GS}}$. More positive $V_{\mathrm{GS}}$ result in lower channel potentials and higher electron energies in the channel, increasing the driving force (kinetics) for electron transfer to the oxidant in the electrolyte (Figure 3 (c)). This presents a conundrum for the amperometric OECT. To ensure stable operation of the OECT, a pseudoreference electrode is utilized as the gate. However, generating too large gate current from the redox reactions $(\sim 1 \mu \mathrm{A})$ results in shifts of gate potential, which defeats the purpose of utilizing such an electrode. Hence, even if the OECT were to be optimized to maximize faradaic reaction currents from the analyte, e.g. by increasing $V_{\mathrm{GS}}$, it would result in deleterious effects on device stability. Furthermore, the faradaic current only emerges above the background current when the OECT is in its off-state as the on-current of an OECT is typically larger than a few $\mu \mathrm{A}$, which restricts the $V_{\mathrm{GS}}$ range of operation. Therefore, this OECT architecture not only does not amplify the reaction current, but it also performs more poorly than the simpler chronoamperometric setup where the absolute reaction current is measured. 
While utilizing a non-polarizable $\mathrm{Ag} / \mathrm{AgCl}$ electrode ensures operational stability, it fundamentally prevents changes in channel potential. Without the change in channel charge density, there can be no change in channel conductivity and hence no amplification of the reaction current. This challenge may be resolved by utilizing a gate electrode with a tunable potential, such as an OMIEC. This material can be the same as that of the channel for fabrication convenience, but our analysis is independent of this choice. While this OECT design may exhibit less reliable performance due to the variability of the gate potential (Figure 2 (d-f)), it is worth investigating as this design has been widely explored as chemical sensors due to their ease in fabrication and their ability to amplify.

Identical measurements were conducted for this OECT with the only difference being the gate material - p(g3T2) instead of an $\mathrm{Ag} / \mathrm{AgCl}$ pellet (SI Figure 2 (c)). While our system involves both gate and channel participating in the oxidation reaction, the following arguments also apply to OECTs where the reaction is selectively limited to either electrode ${ }^{[11]}$ (e.g. biological catalysts selectively immobilized on one of the two electrodes). Due to the application of a fixed $V_{\mathrm{GS}}$, potential changes on the reactive electrode would result in similar potential changes on the other as well. Transfer curves obtained in the OECT's initial state and after addition of $\mathrm{H}_{2} \mathrm{O}_{2}$ (Figure 3 (d)) show a large difference after conducting redox reactions within the electrolyte. The shift in threshold voltage and maximum transconductance to more positive $V_{\text {GS }}$ provides clear evidence for oxidation of both the gate and channel. This observation is further supported by potential measurements on the gate and source electrodes (Figure 3 (e)), where potentials on both gate and source increase by $\sim 200 \mathrm{mV}$ due to their redox-activity. Unlike the previous case where a nonpolarizable gate was used, the channel potential, hence its charge state, is no longer pinned to a fixed value. Instead, it changes in concert with the gate electrode by applying a fixed potential difference, $V_{\mathrm{GS}}$. Figure 3 (f) summarizes the changes in electron energy levels on both the gate and channel before and after addition of a redox analyte. In addition to the change in drain current from changes in charge density, there exists additional potential-dependent reaction currents (red arrows) (see SI Figure 3 for gate currents). While the potential difference between gate and channel is fixed, the position of the Fermi level in the semiconductors and, consequently, the OECT I-V characteristics depend on the reaction currents. Hence, these redox reactions result in a shift in potential and corresponding change in charge density of the OECT channel, enabling the amplification of reaction currents.

However, the amplification is unreliable and this OECT architecture encounters several additional challenges. In the OECT community, it is common to identify the OECT's maximum transconductance in its pristine state (i.e. $V_{\mathrm{GS}}=-0.3 \mathrm{~V}$ ) and operate the device at that predetermined gate voltage for analyte detection. However, the maximum transconductance point moves during operation and thus results in poorly reproducible performance, as seen from the drastic $V_{\mathrm{T}}$ shift due to over-oxidation of the channel (Figure 3 (d) and SI Figure 4). Furthermore, over-oxidation of the channel (SI Figure 1) may lead to irreversible chemical degradation that changes the channel's electrochemical characteristics. Alternatively, from Figure 3 (d), it is tempting to conclude that operating the OECT anywhere between $V_{\mathrm{GS}}=-0.1 \mathrm{~V}$ to $+0.1 \mathrm{~V}$ can result in amplification. However, it is important to note that this $\sim 200 \mathrm{mV}$ shift in $V_{\mathrm{T}}$ corresponds 
to one particular $\mathrm{H}_{2} \mathrm{O}_{2}$ concentration i.e. $33 \mathrm{mM}$. Indeed, different concentrations of $\mathrm{H}_{2} \mathrm{O}_{2}$ result in varying shifts in $V_{\mathrm{T}}$ (SI Figure 5), making the determination of optimal applied $V_{\mathrm{GS}}$ a circular argument of first having to know the concentration of an unknown analyte before measuring it. Furthermore, the oxidation of the gate is irreversible, requiring a new gate electrode for each measurement. The initial potential of the gate may be recovered by applying a potential difference with respect to a standard reference electrode. However, incorporation of this third electrode defeats the purpose of utilizing a simple 2-terminal OECT architecture.

Due to the poor reproducibility of operating at gate voltages above $V_{\mathrm{T}}$ (SI Figure 4), we resorted to operation of the OECT below threshold i.e. $V_{\mathrm{GS}}=+0.3 \mathrm{~V}$. Due to operation in its OFF state, the faradaic current results in negligible changes in channel charge density. Hence, the performance of the OECT then mimics that of its counterpart with $\mathrm{Ag} / \mathrm{AgCl}$ gate (Figure 3 (b)). The OECT with OMIEC gate experiences the same potential-dependent complexities while suffering additional challenges arising from irreversible changes in its transfer curve characteristics. As an amplifier, having variable transfer characteristics is poor device design as it fundamentally changes the amplifying characteristics irreversibly. Furthermore, we note that the peaking transconductance implies that linear shifts in $V_{\mathrm{T}}$ induce non-linear changes in $I_{\mathrm{D}}$. This effect may be less pronounced for small $V_{\mathrm{T}}$ shifts, but this would limit the detectable concentration range of analytes. Alternatively, these non-linear effects can be ameliorated by utilizing transistors with a constant transconductance. However, there has yet to be an OECT with such characteristics operated in aqueous electrolytes. These challenges in conjunction with the irreversible nature of the OMIEC oxidation makes measurement of an unknown concentration extremely challenging as the sensor cannot be calibrated and re-used. 
(a)

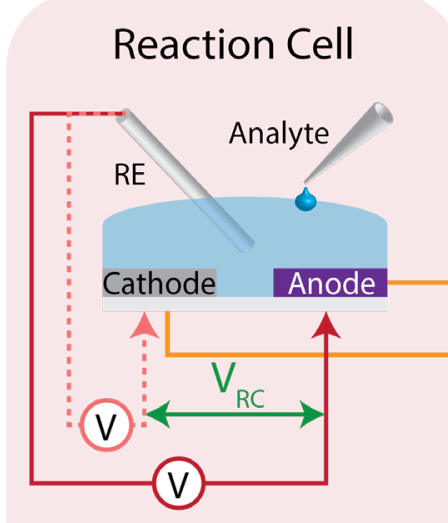

(b)

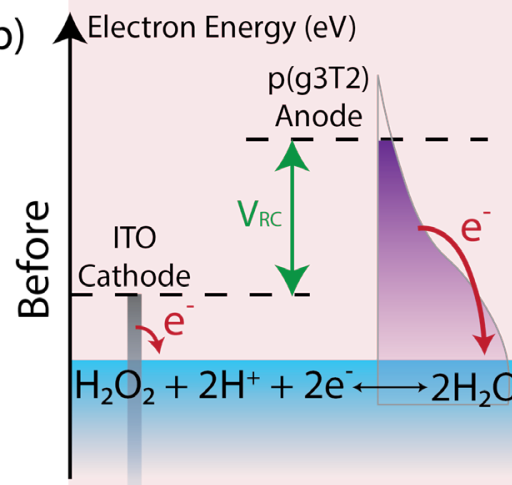

(c)

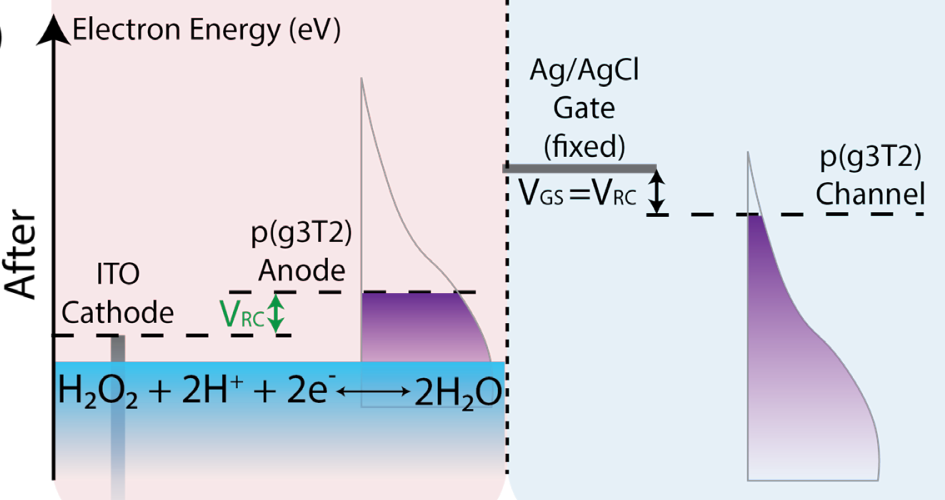

(d)

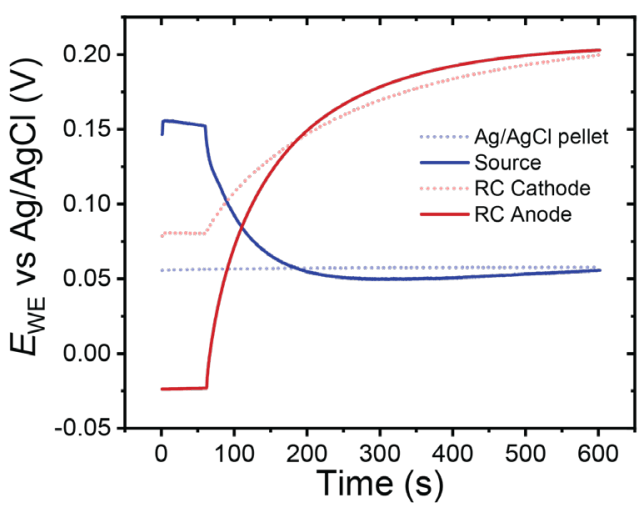

(e)

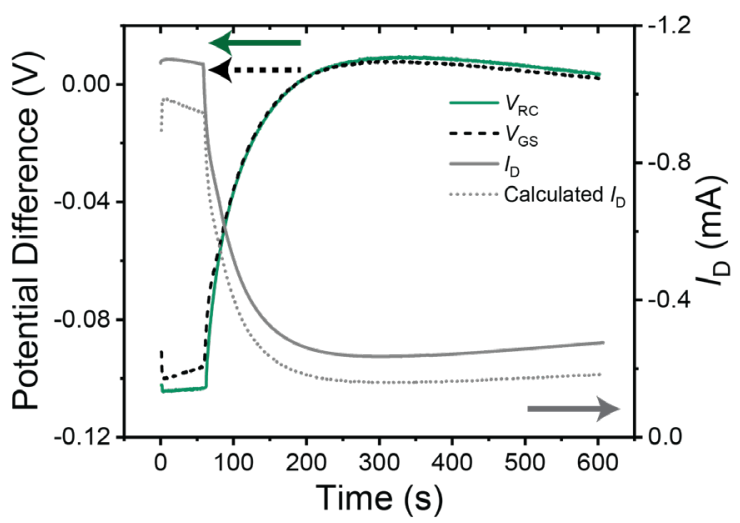

(f)

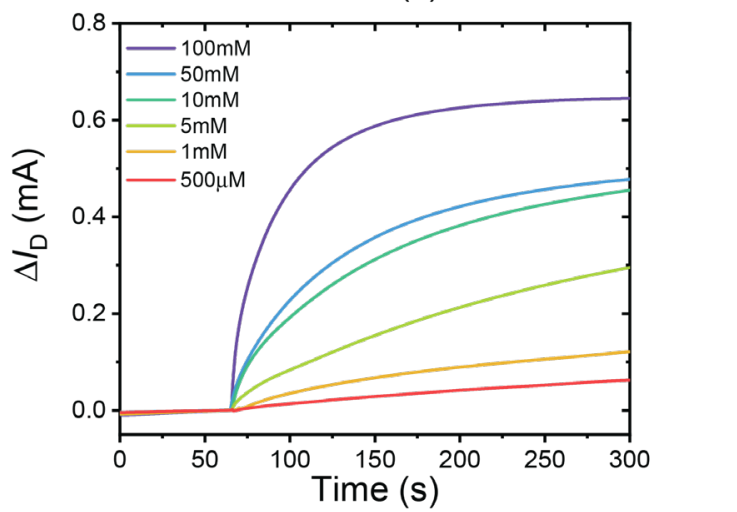

Figure 4. Operation mechanism of a Reaction Cell OECT using an $\mathrm{Ag} / \mathrm{AgCl}$ gate electrode. (a) Schematic of RC and OECT where the RC anode is in electrical contact with the OECT's $\mathrm{Ag} / \mathrm{AgCl}$ gate electrode and the $\mathrm{RC}$ cathode is in electrical contact with the source. Redox analyte is added to RC's electrolyte while conducting potential measurements on the RC's anode, cathode as well as the OECT's gate and source. (b) Schematic of electron energy levels in the RC ITO cathode (grey), $\mathrm{p}(\mathrm{g} 3 \mathrm{~T} 2$ ) anode (purple, left), OECT's $\mathrm{Ag} / \mathrm{AgCl}$ gate electrode (solid line), and OECT p(g3T2) channel (purple, right) in the system's initial state. Electron transfer from RC electrodes to the analyte in the RC electrolyte is shown in red arrows. Difference in energy levels between $\mathrm{RC}$ cathode and anode is indicated by $V_{\mathrm{RC}}$. Due to the fixed potential on the $\mathrm{Ag} / \mathrm{AgCl}$ gate, $V_{\mathrm{GS}}=V_{\mathrm{RC}}$ is imposed on the channel. (c) Change in electron energy levels in the electrode after system has reached a new equilibrium in the presence of the oxidative analyte in the RC. 
Drop in $V_{\mathrm{GS}}=V_{\mathrm{RC}}$ results in filling of the channel valence band, dedoping the OECT channel. (d) Plot of potentials of $\mathrm{Ag} / \mathrm{AgCl}$ gate electrode (blue dashed), OECT source (blue solid), $\mathrm{RC}$ anode (red solid) and RC cathode (red dashed). (e) Plot of potential differences across RC (green) and OECT's gate-source (black dashed) as well as the drain current (grey) and the calculated drain current (grey dashed). (f) RC-OECT calibration characteristics. Magnitude of change in RCOECT drain current over time upon addition of $200 \mu \mathrm{L} \mathrm{H} \mathrm{H}_{2} \mathrm{O}_{2}$ of different concentrations to 400 $\mu \mathrm{L}$ RC PBS electrolyte.

The pitfalls encountered by the amperometric all-in-one OECTs offer important learning points for achieving reliable amplification in a faradaic chemical sensor. Firstly, the OECT as an amplifier should have stable and reproducible characteristics, implying the need for a non-polarizable gate electrode such as $\mathrm{Ag} / \mathrm{AgCl}$. Secondly, to achieve amplification, the transduction mechanism should involve steady-state changes to the charge density of the channel, i.e. its potential. These design principles are inherently contradictory for OECTs that combine both chemical detection and transistor function in the same compartment. To resolve these apparent contrasting principles, the chemical reaction should first be transduced into a potential change that subsequently gates the OECT. Furthermore, the separation of chemical reactions from the OECT compartment prevents complex reactions in the OECT due to the interplay of gate and drain voltages. These insights support the design of the RC-OECT described in our previous work, which amplifies reaction currents by $10^{3}$ with high reproducibility. ${ }^{[22]}$

To understand the performance of the RC-OECT, similar potential measurements were conducted on the $\mathrm{Ag} / \mathrm{AgCl}$ gate and source electrodes of the OECT as well as the ITO cathode and $\mathrm{p}(\mathrm{g} 3 \mathrm{~T} 2)$ anode of the $\mathrm{RC}$ while $\mathrm{H}_{2} \mathrm{O}_{2}$ was added to the RC's electrolyte (Figure 4 (a)). The electrode potentials are plotted in Figure 4 (d). The difference in potential between the anode and cathode is defined as $V_{\mathrm{RC}}$ while the difference between the gate and source is defined as $V_{\mathrm{GS}}$ (Figure 4 (e)). After addition of $\mathrm{H}_{2} \mathrm{O}_{2}$ to the $\mathrm{RC}$ at $60 \mathrm{~s}$, the potentials on both the $\mathrm{RC}$ anode and cathode increase, with the anode increasing by a factor of two more than the cathode. The potential on the $\mathrm{Ag} / \mathrm{AgCl}$ gate pellet remains constant, as expected, hence all the $V_{\mathrm{RC}}$ potential is transferred to the OECT channel. To further verify the RC's effectiveness at gating the OECT, $V_{\mathrm{RC}}$ and $V_{\mathrm{GS}}$ are plotted in Figure 4 (e). $V_{\mathrm{RC}}$ and $V_{\mathrm{GS}}$ are almost identical, indicating that the total change in potential across the RC is dropped across the gate-source of the OECT and since a non-polarizable gate is used, the channel experiences all the potential change. As $V_{\mathrm{RC}}$ is akin to applying an external $V_{\mathrm{GS}}$, the OECT's ability to transduce potential to drain current modulation was further verified by calculating the expected drain current (Figure 4 (e)) based on the OECT's transfer characteristics (SI Figure 6). The calculated drain current agrees closely with the experimental current, indicative that the RC indeed acts as a potentiometric modulator for the OECT. Furthermore, as no chemical reactions are conducted within the OECT and a stable gate electrode is used, the OECT retains its transfer characteristics (SI Figure 6). This allows the OECT to be reused for multiple measurements, while retaining consistent amplification characteristics. Lastly, by designing the RC-OECT to be modular, different RCs can be multiplexed to the same OECT for monitoring different reactions. 
These findings are summarized in two schematics: the flow of charges within the RC-OECT (SI Figure 2 (f)) and changes in electron energy levels of the electrodes (Figure 4 (b and c)). The loss of electrons from the $\mathrm{RC}$ anode to $\mathrm{H}_{2} \mathrm{O}_{2}$ is compensated by the flow of electrons from the $\mathrm{Ag} / \mathrm{AgCl}$ pellet, via oxidation reactions on the gate electrode $\left(\mathrm{Ag} \mathrm{Cl}^{-} \rightarrow \mathrm{AgCl}+e^{-}\right)$. The resultant drop in $\left[\mathrm{Cl}^{-}\right]$in the electrolyte is compensated by the injection of anions from the OECT channel due to the reduction of the channel via electrons injected from the source. This change in charge density on the channel enables large changes in conductivity, thereby amplifying the small reaction currents on the RC anode into large drain current modulations. In Figure 4 (b and c). The cathode and anode have different energy levels initially. Upon addition of the oxidant, electrons are transferred to the analyte, changing potentials on both electrodes, and the overall potential difference between them $\left(V_{\mathrm{RC}}\right)$. This potential difference is then utilized to gate the OECT $\left(V_{\mathrm{RC}}=\right.$ $\left.V_{\mathrm{GS}}\right)$. Due to the virtually infinite reservoir of electrons on the $\mathrm{Ag} / \mathrm{AgCl}$ pellet, its electron energy level remains mostly constant. Hence, all changes occur on the channel where its reduction results in increase in its electron energy levels.

From our earlier analyses of OECTs with different gate electrodes, utilizing a non-polarizable gate electrode is crucial in maintaining reproducible performance. To further test this hypothesis on the RC-OECT architecture, we substituted the $\mathrm{Ag} / \mathrm{AgCl}$ pellet with a $\mathrm{p}(\mathrm{g} 3 \mathrm{~T} 2)$ gate electrode (SI Figure 7). While the RC-OECT is still able to exhibit large drain current modulations and amplification, it suffers from poorer stability and reproducibility. Despite disconnecting the RC from the OECT after measurements, the OECT did not recover its initial characteristics, where the threshold voltage shifted by $\sim 100 \mathrm{mV}$, corresponding to the change in $V_{\mathrm{RC}}=V_{\mathrm{GS}}$. This indicates that using a polarizable or reactive OMIEC gate is not desirable in either potentiometric or amperometric OECT-based sensors. 


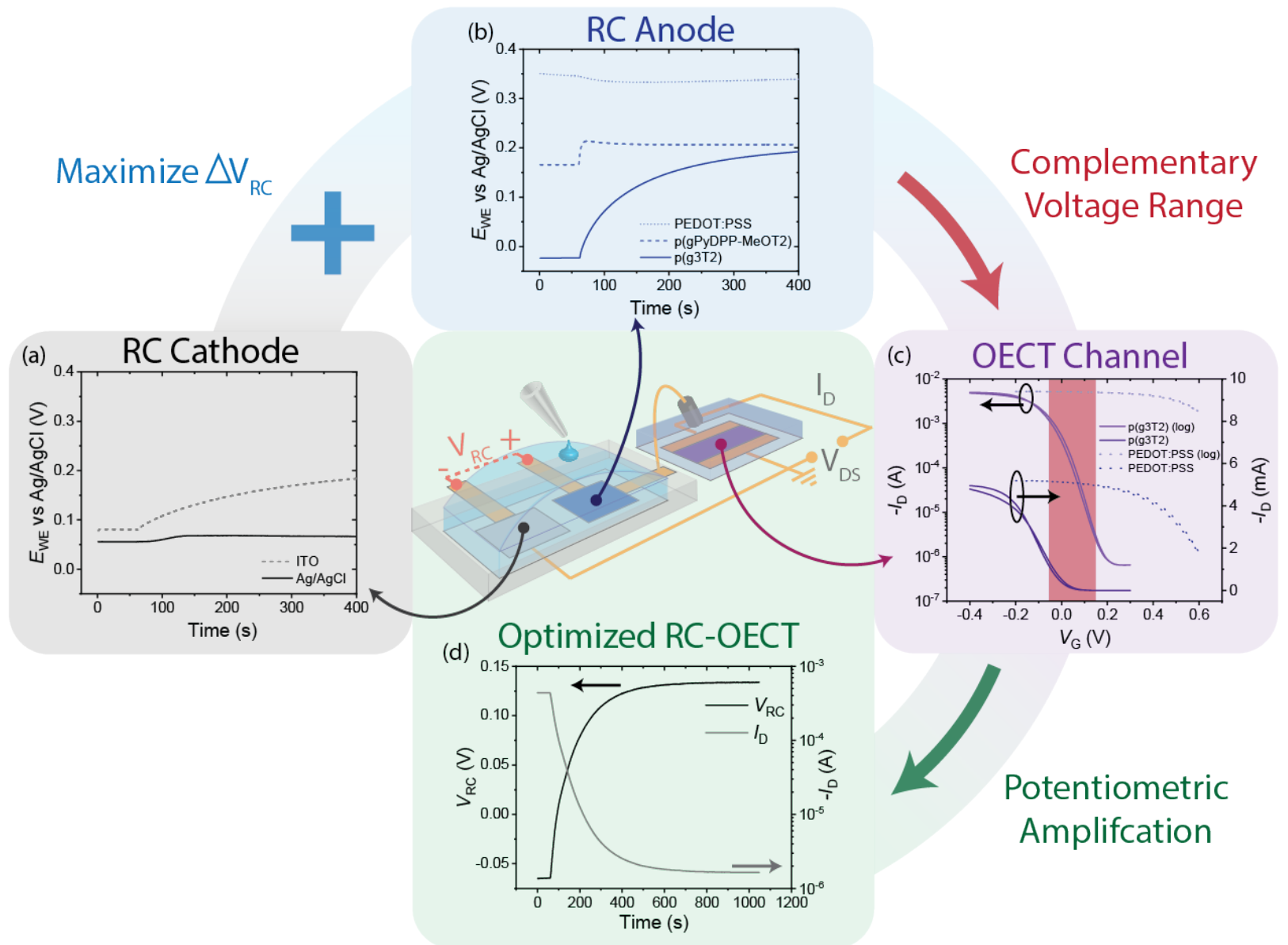

Figure 5. Optimization of RC-OECT. $\Delta V_{\mathrm{RC}}$ is maximized by minimizing oxidation on RC cathode and maximizing oxidation on RC anode. $\Delta I_{\mathrm{D}}$ is maximized by optimizing OECT to exhibit maximum current modulation in complementary voltage range to $\Delta V_{\mathrm{RC}}$. (a) $\mathrm{RC}$ Cathode: Potentials of ITO (grey dashed) and $\mathrm{Ag} / \mathrm{AgCl}$ pellet (black solid) vs. saturated $\mathrm{Ag} / \mathrm{AgCl}$ reference electrode with addition of $\mathrm{H}_{2} \mathrm{O}_{2}$ at $60 \mathrm{~s}$. (b) $\mathrm{RC}$ Anode: Potentials of PEDOT:PSS (lightest blue, dotted), p(gPyDPP-MeOT2) (medium blue, dash), and p(g3T2) (dark blue, solid) vs. saturated $\mathrm{Ag} / \mathrm{AgCl}$ reference electrode with addition of $\mathrm{H}_{2} \mathrm{O}_{2}$ at $60 \mathrm{~s}$. (c) OECT transfer characteristics of $\mathrm{p}\left(\mathrm{g} 3 \mathrm{~T} 2\right.$ ) (purple lines) and PEDOT:PSS. (d) $V_{\mathrm{RC}}$ (black) and $I_{\mathrm{D}}$ (grey) of optimized RC-OECT using $\mathrm{Ag} / \mathrm{AgCl}$ pellet as $\mathrm{RC}$ cathode, $\mathrm{p}(\mathrm{g} 3 \mathrm{~T} 2)$ as $\mathrm{RC}$ anode, and $\mathrm{p}(\mathrm{g} 3 \mathrm{~T} 2)$ as OECT channel.

The separation of chemical transduction and amplification into two compartments enables individual optimization of the RC and OECT. Firstly, $\Delta V_{\mathrm{RC}}$ can be maximized by selecting anode materials that are most sensitive to the redox analyte of interest while the cathode is passive to the analyte. To demonstrate this concept, we utilized an $\mathrm{Ag} / \mathrm{AgCl}$ pellet instead of ITO as the cathode to minimize the cathode's oxidation (Figure 5 (a)). In selecting an anode material, PEDOT:PSS, a common air-sensitive conductive polymer, $\mathrm{p}(\mathrm{gPyDPP}-\mathrm{MeOT} 2),{ }^{[28]}$ a recently developed airstable polymer, and p(g3T2) were investigated. p(g3T2) was selected for the study as it undergoes the largest degree of oxidation among the polymer candidates (Figure 5 (b)). Taking the difference in potentials between the $\mathrm{RC}$ anode and cathode gives a $V_{\mathrm{RC}}$ modulation range of $-0.05 \mathrm{~V}$ to +0.125 
$\mathrm{V}$. Next, to maximize $\Delta I_{\mathrm{D}}$, this range of $V_{\mathrm{RC}}$ should overlap with the range of $V_{\mathrm{GS}}$ where the OECT exhibits maximum transconductance and/or encompass $V_{\mathrm{T}}$. The transfer characteristics of OECTs with p(g3T2) or PEDOT:PSS as the channel material were compared (Figure 5 (c)). While the PEDOT:PSS channel is highly conductive in this $V_{\mathrm{GS}}$ range, its conductivity modulation is a small fraction of its baseline, rendering the measurement of a small $\Delta I_{\mathrm{D}}$ over a large $I_{\mathrm{D}}$ baseline difficult. In contrast, the $\mathrm{p}(\mathrm{g} 3 \mathrm{~T} 2)$ OECT undergoes $\Delta I_{\mathrm{D}} \sim 10^{3}$ in the same $V_{\mathrm{GS}}$ range (Figure 5 (d)). See SI Figure 8 for more details on optimizing channel aspect ratio for maximum transconductance and SI Figure 9 for full characterization of the RC-OECT. As the RC and OECT perform distinct roles as redox to potential transducer and amplifier respectively, RC electrode and OECT channel materials can be selected to fulfill their specific metrics rather than attempting to find compromises between competing design metrics as needed in the all-in-one OECTs.

\section{Discussion}

Unlike solid-state field-effect transistors (FETs), potential differences ( $V_{\mathrm{GS}}$ and $\left.V_{\mathrm{DS}}\right)$ during OECT operation do not provide sufficient information nor control of the phenomena occurring at the OECT's electrodes. Hence, fully utilizing the amplification properties of OECTs for reliable faradaic chemical detection requires more detailed understanding of the potentials and charge density of its electrodes.

Our analysis of electrochemical processes in various OECT architectures shows fundamentally that a non-polarizable gate electrode such as $\mathrm{Ag} / \mathrm{AgCl}$ is imperative to preserve the stability and reproducibility of the OECT's I-V characteristics. However, conducting faradaic reactions within the electrolyte of this OECT does not amplify reaction currents. Conversely, an OMIEC gate, in principle leads to amplification however the resulting shift of the OECT I-V characteristics, which is irreversible in a 2-electrode geometry, makes this geometry fundamentally ill-suited as a sensor. We note that this shift is inevitable as it is inherent to the sensing process. Moreover, the nonconstant transconductance leads to difficulties in sensor calibration, which will be sensitive to the specifics of how transconductance depends on $V_{\mathrm{GS}}$. Finally, the presence of the potentials needed to drive the OECT in the sensing environment leads to parasitic reactions, with molecular oxygen for instance. Any parasitic side reaction on the OECT gate and channel are extremely complex to model due to the asymmetric electric fields within the OECT, exacerbated by the potentialdependence of reaction currents (see Supplemental Information). These additionally lead to further irreversible changes in the OECT characteristics, exacerbating the irreproducibility of the sensing measurements.

To achieve both operational stability and amplification, the reactive OMIEC material is physically separated from the OECT in a Reaction Cell which is utilized to gate an OECT with an $\mathrm{Ag} / \mathrm{AgCl}$ gate electrode. By modulating the OECT channel potentiometrically, the RC fully leverages the excellent transconductance of the OECT, enabling the amplification of minute reaction currents into easily detected drain current modulations. The modularity of the RC-OECT architecture enables designing of the RC and OECT separately, where each component can be optimized for specific metrics of potential change and transconductance, respectively as demonstrated in the optimal materials choice to match sensing range with peak OECT transconductance. This avoids constraints faced by all-in-one OECTs where one material (e.g. channel) has to fulfill multiple 
functions simultaneously while ultimately not being optimized for any. Improving amplification of the RC-OECT requires further maximization of the OECT's transconductance by changing the channel $\mathrm{W} / \mathrm{L}$ aspect ratio, enabling large improvements for small changes to device structure. This, in addition to the ability to select different materials and device geometries for the RC electrodes and OECT, enables great flexibility during RC-OECT device optimization, opening myriad opportunities for device development.

Following the general engineering design principles of minimizing complexity while maximizing performance, the RC-OECT architecture presents an effective approach to OECT-based redox chemical sensors. By demonstrating the importance of understanding the underlying electrochemical phenomena in OECTs to rationalize device design, we envision that these insights allow further systematic development of electrochemical device architectures for chemical sensing. 


\section{References}

[1] B. D. Paulsen, K. Tybrandt, E. Stavrinidou, J. Rivnay, Nat. Mater. 2020, 19, 13.

[2] A. Giovannitti, I. P. Maria, D. Hanifi, M. J. Donahue, D. Bryant, K. J. Barth, B. E. Makdah, A. Savva, D. Moia, M. Zetek, P. R. F. Barnes, O. G. Reid, S. Inal, G. Rumbles, G. G. Malliaras, J. Nelson, J. Rivnay, I. McCulloch, Chem. Mater. 2018, 30, 2945.

[3] A. Giovannitti, D. T. Sbircea, S. Inal, C. B. Nielsen, E. Bandiello, D. A. Hanifi, M. Sessolo, G. G. Malliaras, I. McCulloch, J. Rivnay, Proc. Natl. Acad. Sci. U. S. A. 2016, $113,12017$.

[4] M. Moser, T. C. Hidalgo, J. Surgailis, J. Gladisch, S. Ghosh, R. Sheelamanthula, Q. Thiburce, A. Giovannitti, A. Salleo, N. Gasparini, A. Wadsworth, I. Zozoulenko, M. Berggren, E. Stavrinidou, S. Inal, I. McCulloch, Adv. Mater. 2020, 2002748, 1.

[5] J. Gladisch, E. Stavrinidou, S. Ghosh, A. Giovannitti, M. Moser, I. Zozoulenko, I. Mcculloch, M. Berggren, 2019, 1901144, DOI 10.1002/advs.201901144.

[6] J. Mei, Z. Bao, Chem. Mater. 2014, 26, 604.

[7] M. Moser, L. R. Savagian, A. Savva, M. Matta, J. F. Ponder, T. C. Hidalgo, D. Ohayon, R. Hallani, M. Reisjalali, A. Troisi, A. Wadsworth, J. R. Reynolds, S. Inal, I. McCulloch, Chem. Mater. 2020, DOI 10.1021/acs.chemmater.0c02041.

[8] D. S. Bindra, G. S. Wilson, Anal. Chem. 1989, 61, 2566.

[9] J. Rivnay, S. Inal, A. Salleo, R. M. Owens, M. Berggren, G. G. Malliaras, Nat. Rev. Mater. 2018, 3, DOI 10.1038/natrevmats.2017.86.

[10] A. M. Pappa, V. F. Curto, M. Braendlein, X. Strakosas, M. J. Donahue, M. Fiocchi, G. G. Malliaras, R. M. Owens, Adv. Healthc. Mater. 2016, 5, 2295.

[11] G. Méhes, A. Roy, X. Strakosas, M. Berggren, E. Stavrinidou, D. T. Simon, Adv. Sci. 2020, 2000641,1 .

[12] S. Inal, G. G. Malliaras, J. Rivnay, Nat. Commun. 2017, 8, 1.

[13] D. A. Bernards, G. G. Malliaras, Adv. Funct. Mater. 2007, 17, 3538.

[14] J. T. Friedlein, R. R. McLeod, J. Rivnay, Org. Electron. physics, Mater. Appl. 2018, 63, 398.

[15] J. Rivnay, P. Leleux, M. Ferro, M. Sessolo, A. Williamson, D. A. Koutsouras, D. Khodagholy, M. Ramuz, X. Strakosas, R. M. Owens, C. Benar, J. M. Badier, C. Bernard, G. G. Malliaras, Sci. Adv. 2015, 1,1 .

[16] D. Khodagholy, J. Rivnay, M. Sessolo, M. Gurfinkel, P. Leleux, L. H. Jimison, E. Stavrinidou, T. Herve, S. Sanaur, R. M. Owens, G. G. Malliaras, Nat. Commun. 2013, 4, 1.

[17] J. Rivnay, P. Leleux, M. Sessolo, D. Khodagholy, T. Hervé, M. Fiocchi, G. G. Malliaras, Adv. Mater. 2013, 25, 7010 . 
[18] D. A. Bernards, D. J. MacAya, M. Nikolou, J. A. Defranco, S. Takamatsu, G. G. Malliaras, J. Mater. Chem. 2008, 18, 116.

[19] D. Ohayon, G. Nikiforidis, A. Savva, A. Giugni, S. Wustoni, T. Palanisamy, X. Chen, I. P. Maria, E. Di Fabrizio, P. M. F. J. Costa, I. Mcculloch, S. Inal, Nat. Mater. 2019, DOI 10.1038/s41563-019-0556-4.

[20] A. M. Pappa, D. Ohayon, A. Giovannitti, I. P. Maria, A. Savva, I. Uguz, J. Rivnay, I. McCulloch, R. M. Owens, S. Inal, Sci. Adv. 2018, 4, DOI 10.1126/sciadv.aat0911.

[21] M. Braendlein, A.-M. Pappa, M. Ferro, A. Lopresti, C. Acquaviva, E. Mamessier, G. G. Malliaras, R. M. Owens, Adv. Mater. 2017, 29, 1605744.

[22] S. T. M. Tan, A. Giovannitti, A. Melianas, M. Moser, B. L. Cotts, D. Singh, I. McCulloch, A. Salleo, Adv. Funct. Mater. 2021, 2010868, 1.

[23] E. Macchia, R. A. Picca, K. Manoli, C. Di Franco, D. Blasi, L. Sarcina, N. Ditaranto, N. Cioffi, R. Österbacka, G. Scamarcio, F. Torricelli, L. Torsi, Mater. Horizons 2020, DOI 10.1039/c9mh01544b.

[24] S. Lai, F. A. Viola, P. Cosseddu, A. Bonfiglio, Sensors (Switzerland) 2018, 18, 1.

[25] S. P. White, K. D. Dorfman, C. D. Frisbie, Anal. Chem. 2015, 87, 1861.

[26] D. Moia, A. Giovannitti, A. A. Szumska, I. P. Maria, E. Rezasoltani, M. Sachs, M. Schnurr, P. R. F. Barnes, I. McCulloch, J. Nelson, Energy Environ. Sci. 2019, 1349.

[27] S. E. Doris, A. Pierre, R. A. Street, Adv. Mater. 2018, 30, 1.

[28] A. Giovannitti, R. B. Rashid, Q. Thiburce, B. D. Paulsen, C. Cendra, K. Thorley, D. Moia, J. T. Mefford, D. Hanifi, D. Weiyuan, M. Moser, A. Salleo, J. Nelson, I. McCulloch, J. Rivnay, Adv. Mater. 2020, 1908047, 1. 


\section{Materials and Methods}

OECT fabrication: Photolithographically patterned chips with gold contacts and patterned Parylene to expose regions for the channel were used as OECT chips. p(g3T2) was dissolved in chloroform at $10 \mathrm{mg} / \mathrm{mL}$ and spin coated at $1000 \mathrm{rpm}$, annealed at $60^{\circ} \mathrm{C}$ for 10 minutes, followed by parylene lift-off to form the channel. The OECT device dimension are $L=10 \mu \mathrm{m}, W=2 \mathrm{~mm}$, $\mathrm{d}=120 \mathrm{~nm}$ and operated at $V_{\mathrm{D}}=-0.1 \mathrm{~V}$ for both the amperometric OECT and RC[p(g3T2)/ITO OECT $[\mathrm{p}(\mathrm{g} 3 \mathrm{~T} 2)]$.

RC fabrication: $\mathrm{p}(3 \mathrm{gT} 2)$ was dissolved in chloroform at $10 \mathrm{mg} / \mathrm{mL}$ and spin coated on ITO/glass at $1000 \mathrm{rpm}$, and annealed at $60^{\circ} \mathrm{C}$ for 10 minutes in ambient conditions to yield film thickness of $120 \mathrm{~nm}$.

OECT measurements: Transfer and output characteristics of the OECTs were obtained using a custom LabVIEW program with a K2612 Keithley. Phosphate buffered saline solution was used as the electrolyte and $\mathrm{Ag} / \mathrm{AgCl}$ pellet as gate.

Potential and current measurements: Open circuit measurements were conducted using a Biologic $\mathrm{SP}-300$ with a saturated $\mathrm{Ag} / \mathrm{AgCl}$ reference electrode (BASi). A-OECT: PDMS wells were adhered to the OECT substrate to confine the electrolyte (Phosphate Buffered Saline solution) with either an $\mathrm{Ag} / \mathrm{AgCl}$ pellet or dropcast $\mathrm{p}(\mathrm{g} 3 \mathrm{~T} 2)$ on conductive carbon paper electrode as gate. The reference electrode was immersed in the same electrolyte and the working electrode connected to the pellet while the counter electrode was connected to the source. At the same time, the OECT was operated using a custom LabVIEW program with a K2612 Keithley to impose $V_{\text {GS }}$ and $V_{\mathrm{DS}}$ and to measurement drain and gate currents. A constant drain voltage $\left(V_{\mathrm{D}}=-0.1 \mathrm{~V}\right)$ and gate voltage $\left(V_{\mathrm{G}}\right.$ ranging from $-0.3 \mathrm{~V}$ to $+0.3 \mathrm{~V}$ ) was applied. The drain and gate currents were measured simultaneously after addition of $100 \mu \mathrm{L} 100 \mathrm{mM} \mathrm{H}_{2} \mathrm{O}_{2}$ to $200 \mu \mathrm{L}$ of PBS electrolyte. 
$R C-O E C T$ : The $V_{\mathrm{D}}$ across the OECT was supplied by a $\mathrm{K} 2612$ Keithley and $I_{\mathrm{D}}$ measured using a custom LabVIEW program. The reference electrode was immersed in the RC or OECT electrolytes and the working electrode was connected to the $\mathrm{RC}$ anode or $\mathrm{Ag} / \mathrm{AgCl}$ pellet as well as the $\mathrm{RC}$ cathode and OECT source. $400 \mu \mathrm{L}$ of Phosphate Buffered Saline solution was drop cast on the surface of the reaction cell and $200 \mu \mathrm{L}$ of $100 \mathrm{mM} \mathrm{H}_{2} \mathrm{O}_{2}$ added after 1 minute.

Chronoamperometry measurements: Potential of the working electrode was held at $+0.3 \mathrm{~V}, 0 \mathrm{~V}$, $0.1 \mathrm{~V}$, and $-0.3 \mathrm{~V}$ vs. a saturated $\mathrm{Ag} / \mathrm{AgCl}$ reference electrode and the current flow into the film measured after $200 \mu \mathrm{L}$ of $100 \mathrm{mM} \mathrm{H}_{2} \mathrm{O}_{2}$ to $400 \mu \mathrm{L}$ PBS solution.

Cyclic Voltammetry: The scan rate for the cyclic-voltammogram measurements was $50 \mathrm{mV} / \mathrm{s}$ and conducted in phosphate buffered saline solution in ambient conditions unless stated otherwise. 


\section{Supplementary Information}

(a)

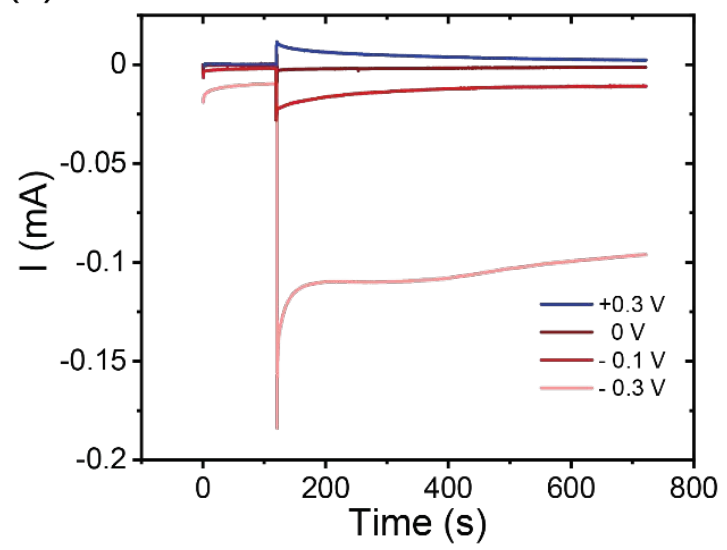

(b)

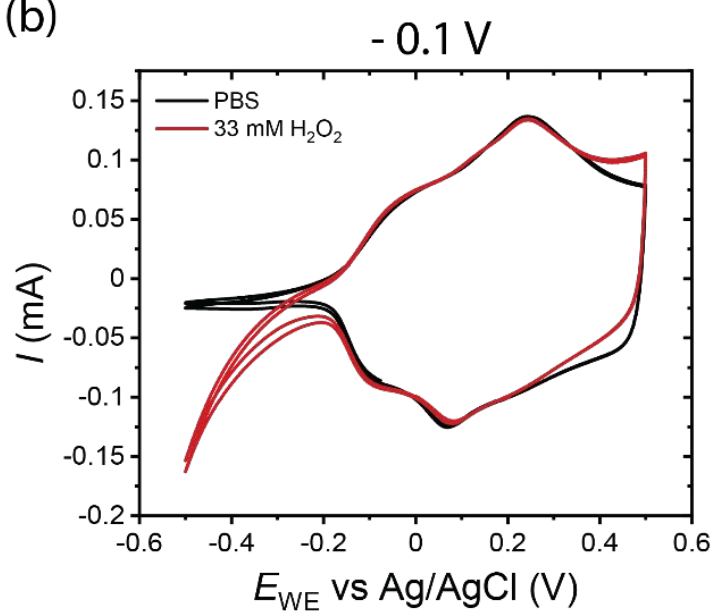

(c)

$-0.3 \mathrm{~V}$

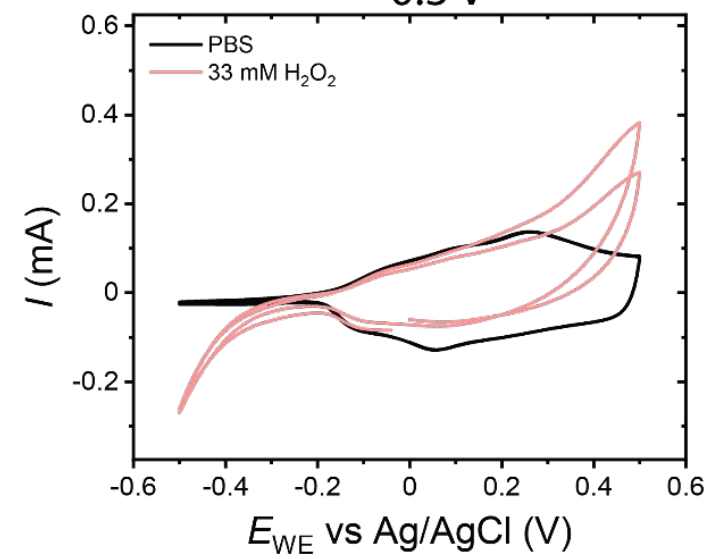

SI Figure 1. Effect of different applied voltages on a p(g3T2) electrode exposed to $\mathrm{H}_{2} \mathrm{O}_{2}$. (a) Chronoamperometric measurements of a spin coated film of $\mathrm{p}(\mathrm{g} 3 \mathrm{~T} 2)$ on ITO/glass held at voltages of $+0.3 \mathrm{~V}, 0 \mathrm{~V},-0.1 \mathrm{~V}$ and $-0.3 \mathrm{~V}$ vs. a saturated $\mathrm{Ag} / \mathrm{AgCl}$ reference electrode. $33 \mathrm{mM} \mathrm{H}_{2} \mathrm{O}_{2}$ is added at $60 \mathrm{~s}$. (b) Cyclic voltammetry of p(g3T2) film in its pristine state in PBS (black) and after 
chronoamperometry at $-0.1 \mathrm{~V}$ and addition of $\mathrm{H}_{2} \mathrm{O}_{2}$. (c) Cyclic voltammetry of $\mathrm{p}(\mathrm{g} 3 \mathrm{~T} 2)$ film in its pristine state in PBS (black) and after chronoamperometry at $-0.3 \mathrm{~V}$ and addition of $\mathrm{H}_{2} \mathrm{O}_{2}$.

It is important to understand the effect of electrode potential on the rate of redox reactions (faradaic currents) for amperometric OECTs. Applying a gate voltage results in changes in channel potential which modulates the channel's ability to undergo redox reactions. Furthermore, the application of a drain voltage across the channel results in asymmetry in potentials across the channel.

To investigate the effect of electrode potentials on reaction currents, chronoamperometry was conducted on $\mathrm{p}\left(\mathrm{g} 3 \mathrm{~T} 2\right.$ ) electrodes in the presence of $\mathrm{H}_{2} \mathrm{O}_{2}$. Potentials are determined in related to a saturated $\mathrm{Ag} / \mathrm{AgCl}$ reference electrode. Different working electrode potentials were applied while current was measured.

Different potentials applied on the working electrode changes the initial energy level of electrons in the OMIEC electrode (degree of filling of the HOMO). The more negative potentials applied on the electrode, the more reduced the OMIEC and the higher the energy of these electrons. Hence, the driving force for electron transfer from the OMIEC electrode to the redox analyte in the electrolyte increases with more negative applied potentials (SI Figure 1 (a)). Applying too extreme potentials also results in degradation of the material as seen by the change in the CV after imposing - 0.3V (SI Figure 1 (c)).

OECT drain voltages are usually on the order of $100 \mathrm{mV}$. In amperometric OECTs where redox analytes are added to the electrolyte, the potential differences across the channel can result in drastic differences in reaction rates. The difference in faradaic reaction current between $0 \mathrm{~V}$ and $0.1 \mathrm{~V}$ is an order of magnitude (from $1 \mathrm{uA}$ to $10 \mathrm{uA}$ ). Hence, applying a relatively small drain voltage of $100 \mathrm{mV}$ results in great differences in faradaic currents on the source versus the drain. Hence, in addition to the potential determined by the gate voltage, it is also important to account for the effect of drain voltage on reaction rates.

In amperometric OECTs with an OMIEC gate, more complexities arise as there is no control over the potential of the gate or channel (only their potential difference). Hence, depending on the operation and processing history of the gate, the potentials on both the gate and channel can change. Furthermore, applying a gate voltage changes the relative reactivity of both the gate and channel. 
(a)

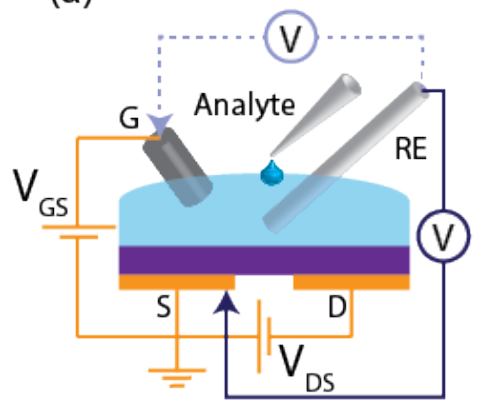

(c)

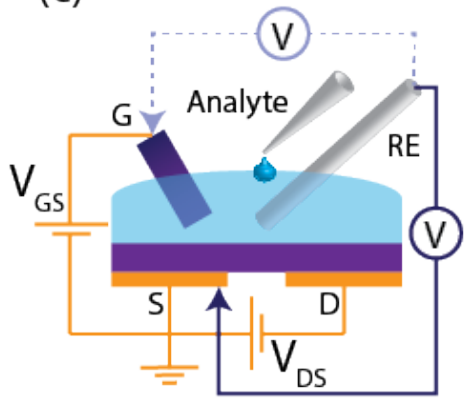

(b)

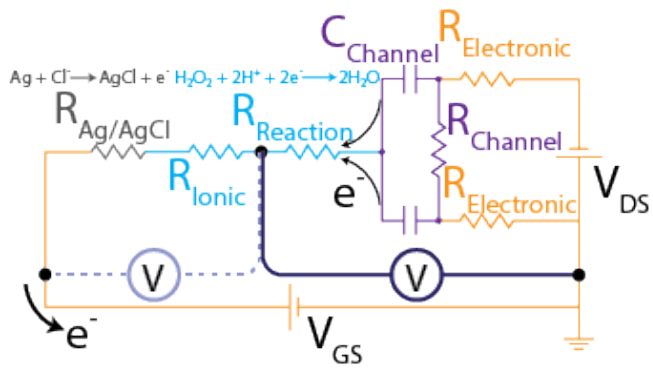

(d)

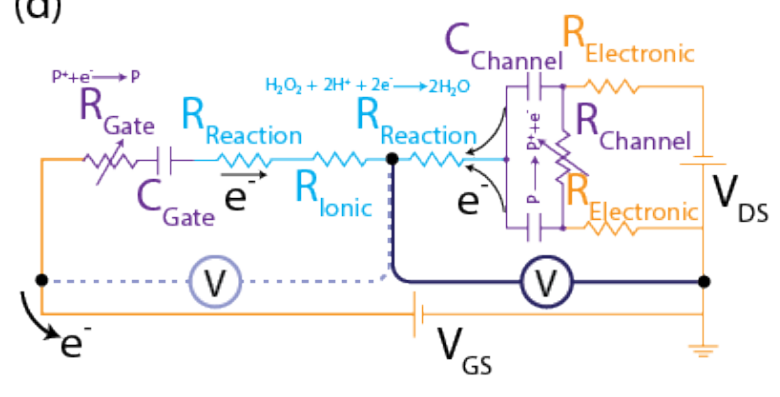

(e)

(f)

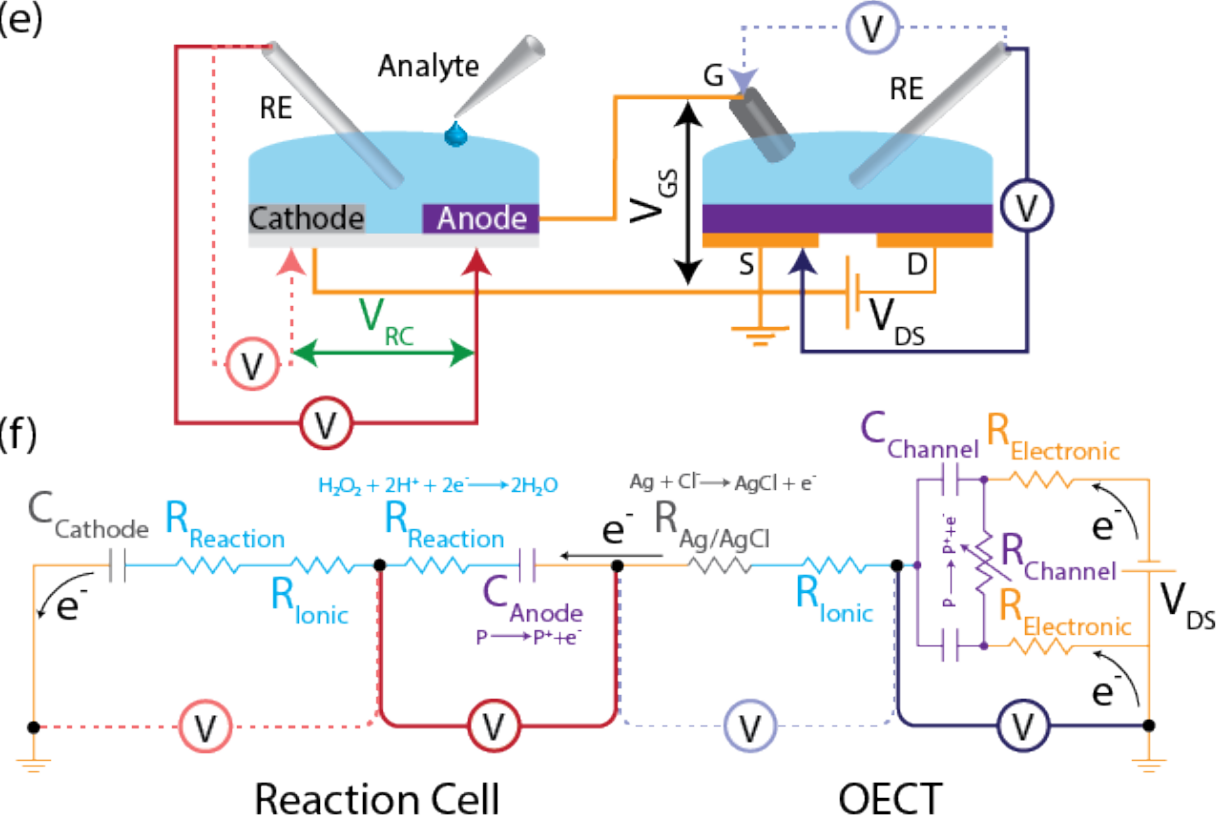

(V)

SI Figure 2. Schematics of potential measurement of electrodes in different OECT architectures and their corresponding equivalent circuits. (a) Schematic of amperometric OECT with $\mathrm{Ag} / \mathrm{AgCl}$ gate showing addition of redox analyte to electrolyte while conducting potential measurements of the $\mathrm{Ag} / \mathrm{AgCl}$ gate and source of the channel. (b) Equivalent circuit of amperometric OECT with $\mathrm{Ag} / \mathrm{AgCl}$ gate showing flow of electrons as result of chemical redox reaction on the channel. (c) Schematic of amperometric OECT with OMIEC gate showing addition of redox analyte to electrolyte while conducting potential measurements of the OMIEC gate and source of the channel. (d) Equivalent circuit of amperometric OECT with $\mathrm{Ag} / \mathrm{AgCl}$ gate showing 
flow of electrons as result of chemical redox reaction on the channel and gate. (e) Schematic of $\mathrm{RC}$ and OECT where the RC anode is in electrical contact with the OECT's $\mathrm{Ag} / \mathrm{AgCl}$ gate electrode and the RC cathode is in electrical contact with the source. Redox analyte is added to RC's electrolyte while conducting potential measurements on the RC's anode, cathode as well as the OECT's gate and source. (f) Sketch of equivalent circuit of RC-OECT showing the oxidation reaction primarily on the anode and the flow of electrons from the OECT to the RC. 


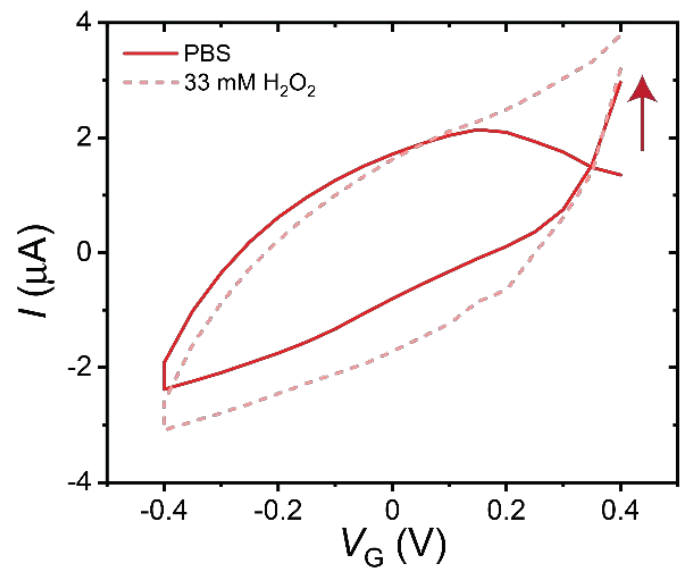

SI Figure 3. Gate current of Amperometric OECT (with OMIE gate) during a transfer curve measurement. Solid line: Prisitine state in PBS. Dashed line: After addition of $33 \mathrm{mM} \mathrm{H}_{2} \mathrm{O}_{2}$ to OECT electrolyte. Red arrow shows larger reaction currents at more positive gate voltages.

(a)

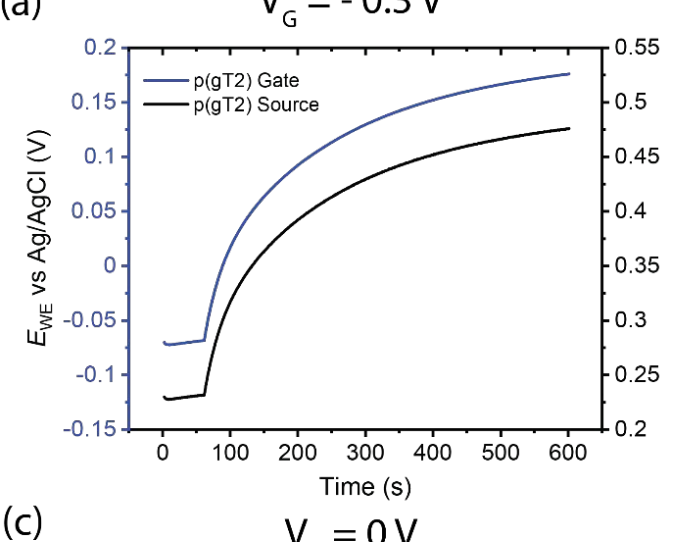

(c)

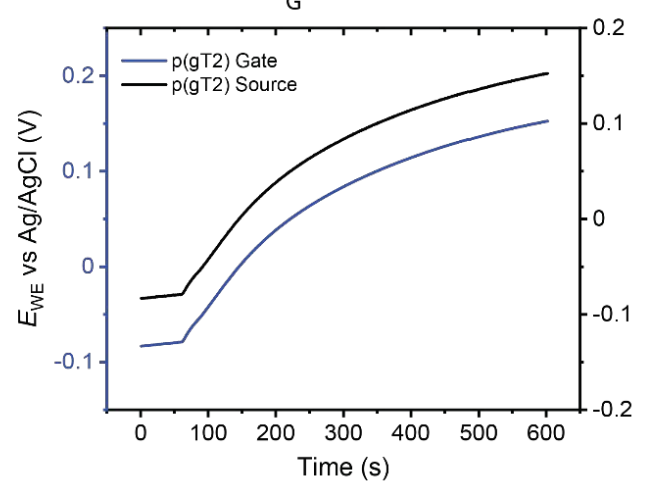

(b)

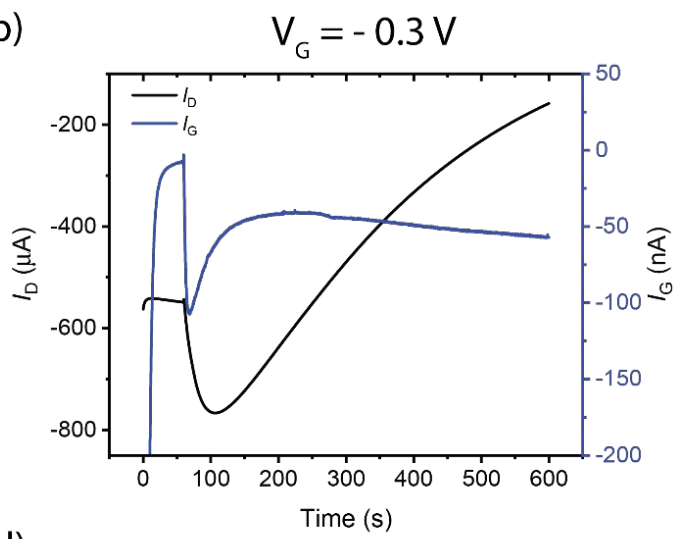

(d)

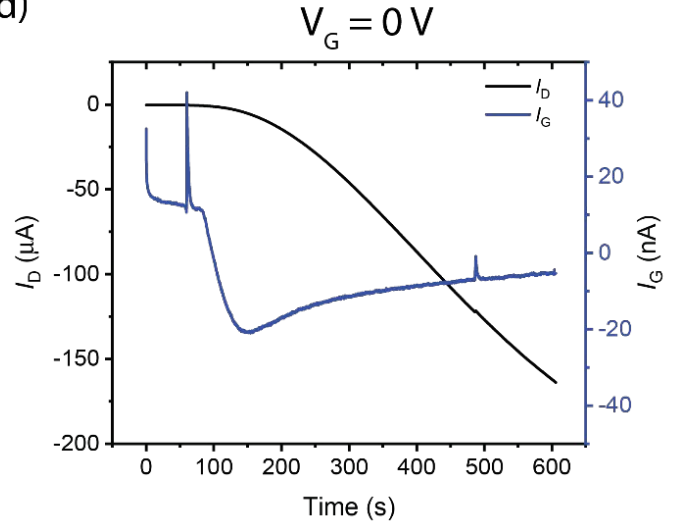

SI Figure 4. Operation of A-OECT (OMIEC gate) at different gate voltages and addition of

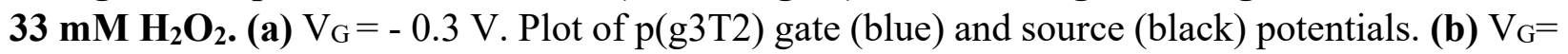
- $0.3 \mathrm{~V}$. Plot of gate (blue) and drain (black) current. a) $\mathrm{V}_{\mathrm{G}}=0 \mathrm{~V}$. Plot of $\mathrm{p}(\mathrm{g} 3 \mathrm{~T} 2$ ) gate (blue) and source (black) potentials. (b) $\mathrm{V}_{\mathrm{G}}=0 \mathrm{~V}$. Plot of gate (blue) and drain (black) current. 


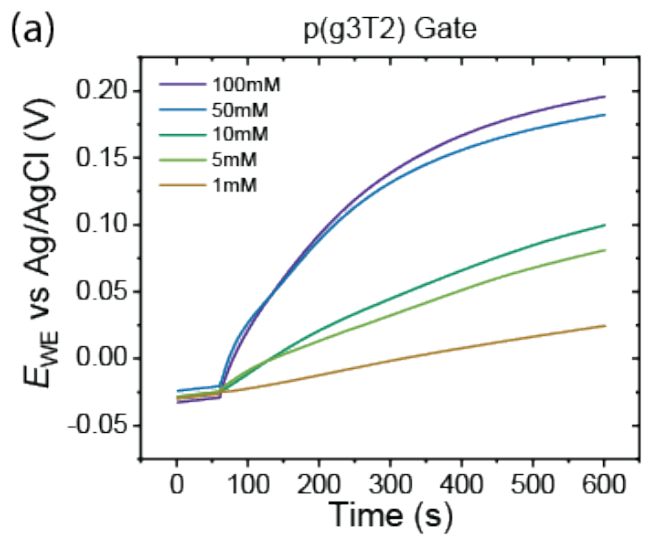

(b)
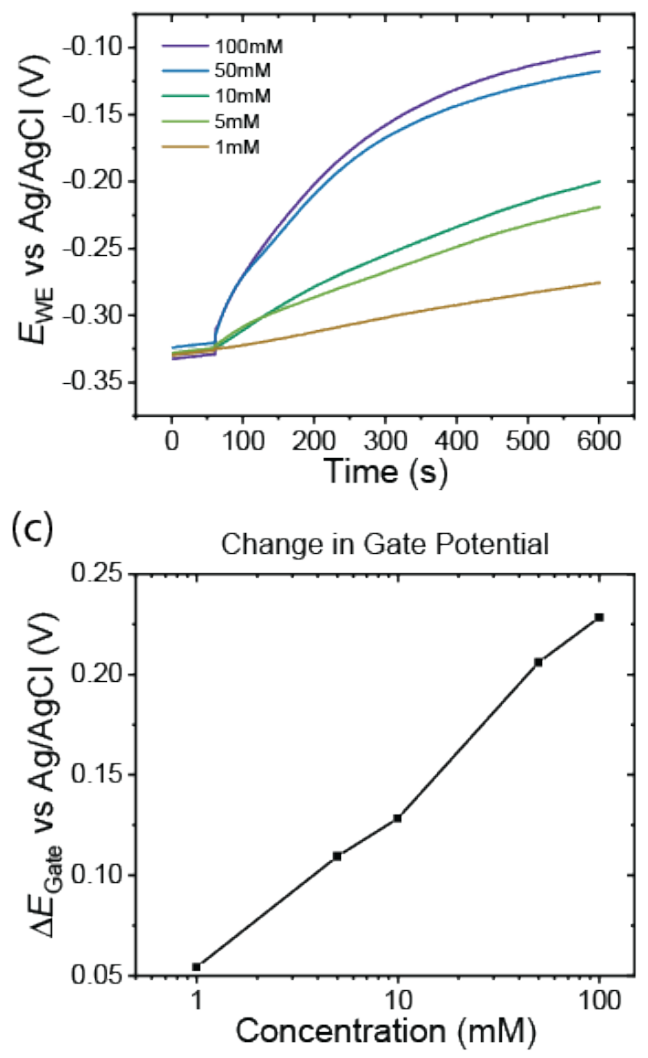

(d)

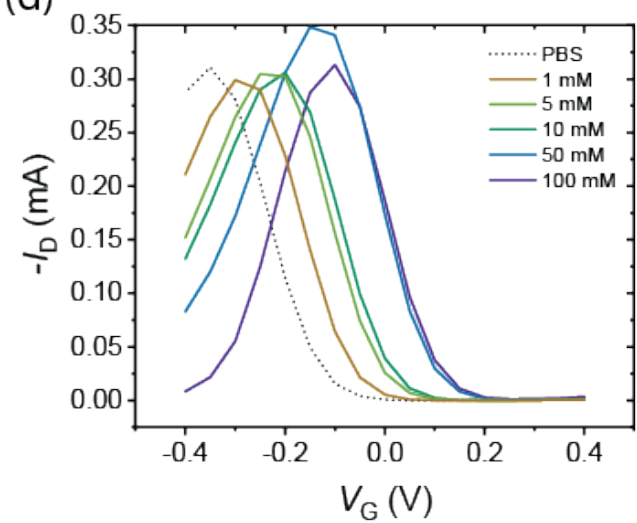

(e)
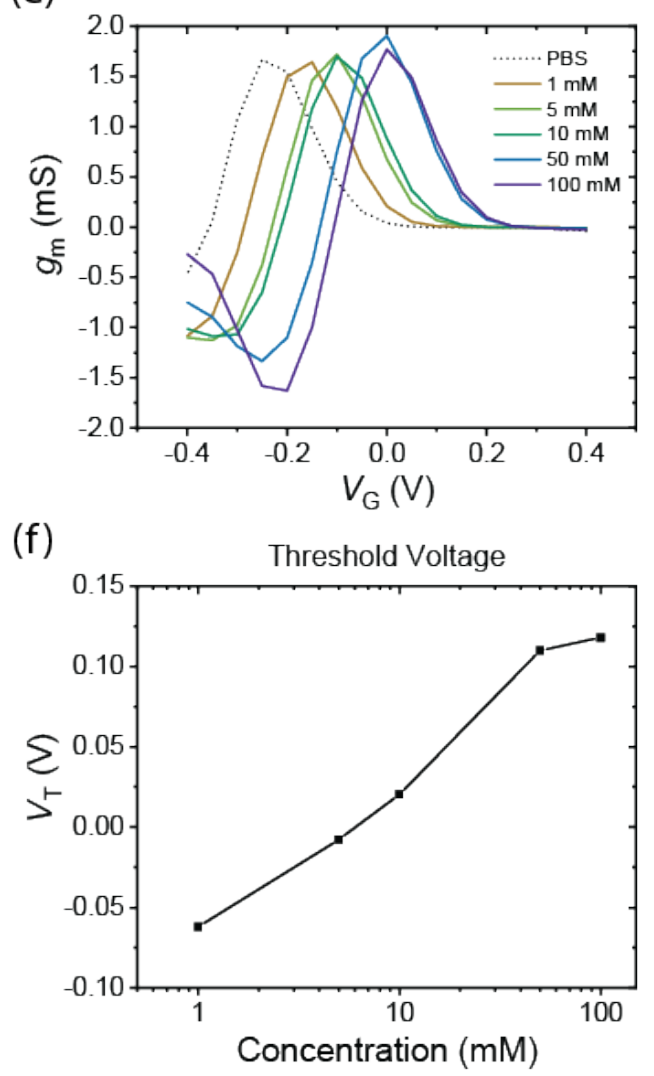

SI Figure 5. Amperometric OECT with OMIEC gate: effect of different concentrations of $\mathbf{H}_{2} \mathbf{O}_{2}$. (a) Measurement of potential on the $\mathrm{p}(\mathrm{g} 3 \mathrm{~T} 2)$ gate with $\mathrm{V}_{\mathrm{G}}=+0.3 \mathrm{~V}$. Different concentrations of $100 \mu \mathrm{L} \mathrm{H}_{2} \mathrm{O}_{2}$ are added to $200 \mu \mathrm{L}$ of PBS OECT electrolyte at 60 s. (b) Concurrent measurement of potential on the source. (c) Plot of change in gate potential vs $\mathrm{H}_{2} \mathrm{O}_{2}$ concentration. (d)Transfer curves of the OECT in its initial state (PBS electrolyte) and after addition of $\mathrm{H}_{2} \mathrm{O}_{2}$ of various concentrations. (e) Corresponding transconductance of OECT in its initial state (PBS electrolyte) and after addition of $\mathrm{H}_{2} \mathrm{O}_{2}$ of various concentrations. (f) Plot of OECT threshold voltage vs $\mathrm{H}_{2} \mathrm{O}_{2}$ concentration. 


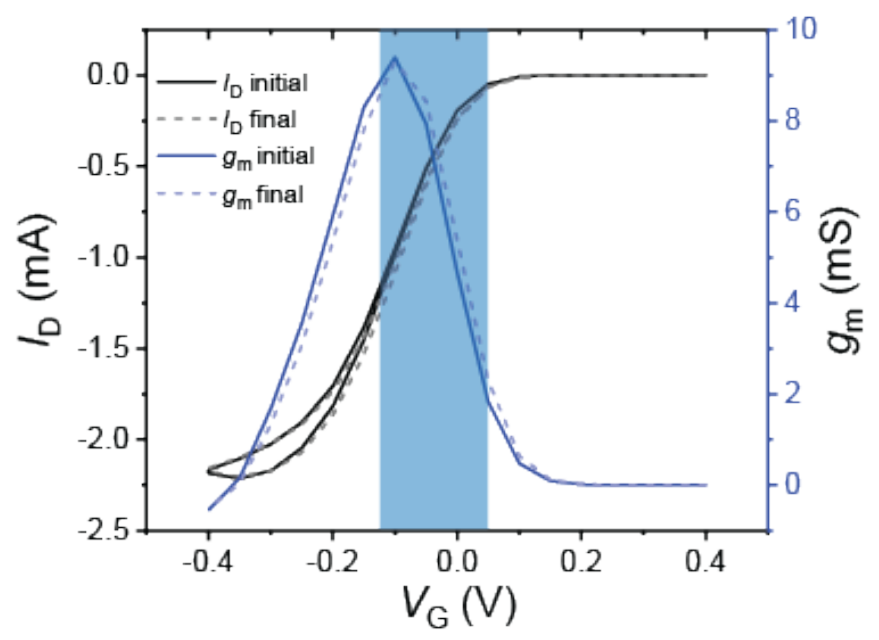

SI Figure 6. Transfer curve (black) and transconductance (blue) of the OECT in its initial state (solid traces) and after addition of the redox analyte to the electrolyte (dashed). Blue rectangle indicate range of $V_{\mathrm{RC}}$.

Stability of OECT before and after RC-OECT operation allows the same OECT to be utilized for multiple reactions by changing $\mathrm{RCs}$. 
(a)

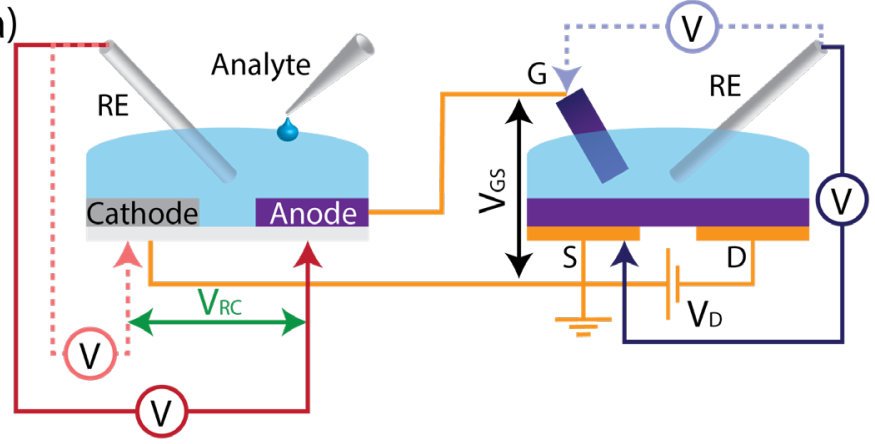

(c)

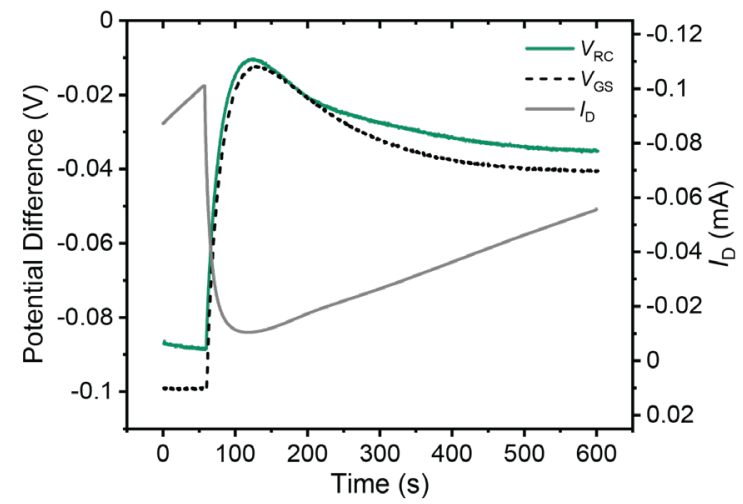

(b)

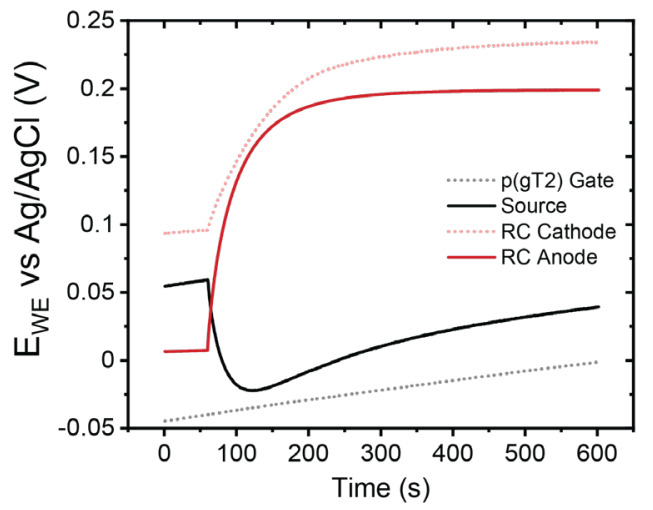

(d)

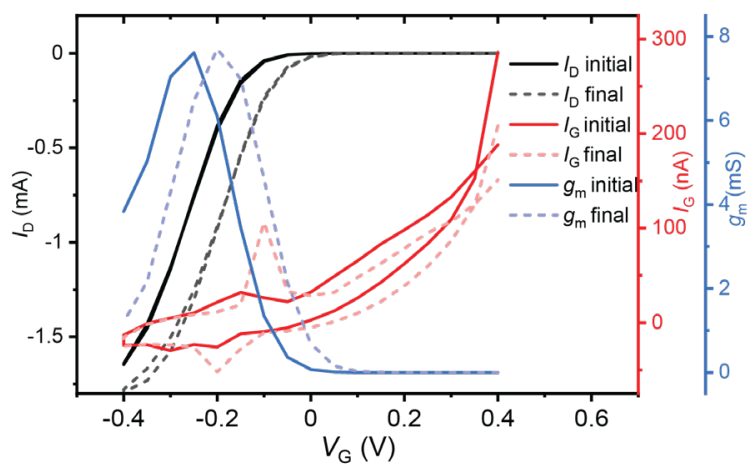

SI Figure 7. Reaction Cell OECT with OMIEC gate electrode in OECT. (a) Schematic of RC and OECT where the RC anode is in electrical contact with the OECT's OMIEC gate electrode and the RC cathode is in electrical contact with the source. Redox analyte is added to RC's electrolyte while conducting potential measurements on the RC's anode, cathode as well as the OECT's gate and source. (b) Plot of potentials of OMIEC gate electrode (grey dashed), OECT source (grey solid), RC anode (red solid) and RC cathode (red dashed). (c) Plot of potential differences across RC (green) and OECT's gate-source (black dashed) as well as the drain current (grey) and the calculated drain current (grey dashed). (d) Transfer curve (black), gate currents (red) and transconductance (blue) of the OECT in its initial state (solid traces) and after addition of the redox analyte to the electrolyte (dashed). 


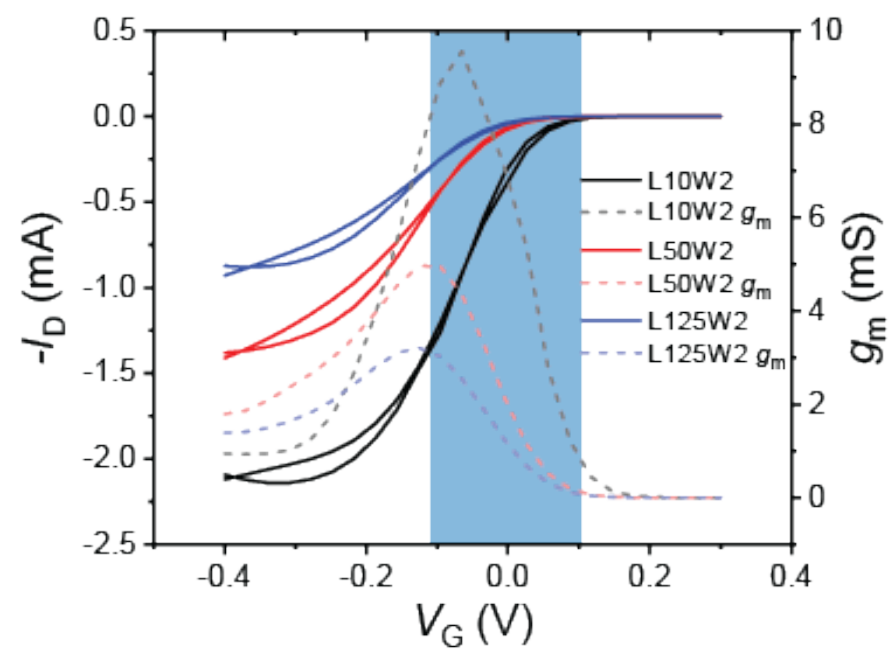

SI Figure 8. Transfer curves (dark solid lines) and transconductances (light dashed lines) of OECTs $W=2000 \mu \mathrm{m}, L=10 \mu \mathrm{m}$ (black), $50 \mu \mathrm{m}$ (red), $125 \mu \mathrm{m}$ (blue), $d=120 \mathrm{~nm}$ operated at $V_{\mathrm{D}}=-0.1 \mathrm{~V}$.

Increasing the $\mathrm{W} / \mathrm{L}$ aspect ratio of the OECT channel increases its transconductance and shifts the maximum transconductance to $V_{\mathrm{GS}}$ values closer to $0 \mathrm{~V}$. Hence, the shortest channel OECT $(\boldsymbol{W}=$ $\mathbf{2 0 0 0} \boldsymbol{\mu m}, L=10 \mu \mathrm{m}, \boldsymbol{d}=\mathbf{1 2 0} \mathbf{~ n m}$ ) geometry was selected for this study. 

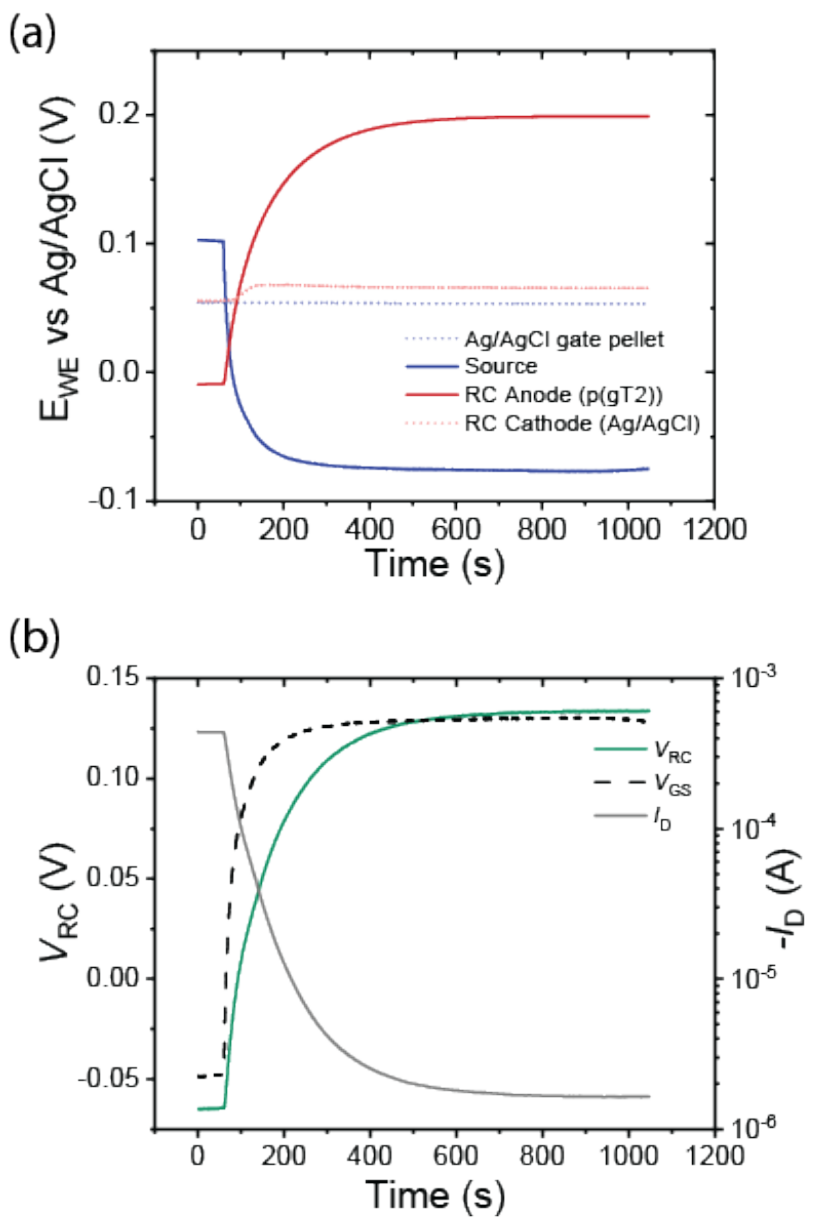

SI Figure 9. Effect of changing RC cathode to $\mathrm{Ag} / \mathrm{AgCl}$ pellet. (a) Plot of potentials of $\mathrm{Ag} / \mathrm{AgCl}$ gate electrode (grey dashed), OECT source (grey solid), RC p(g3T2) anode (red solid) and RC $\mathrm{Ag} / \mathrm{AgCl}$ cathode (red dashed). (b) Plot of potential differences across RC (green) and OECT's gate-source (black dashed) as well as the drain current (grey). 
(a)

$$
\text { Potential ( } \mathrm{V})
$$

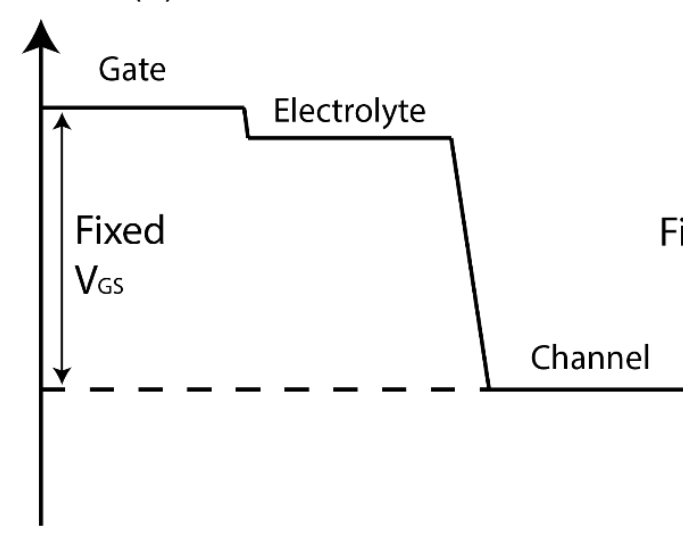

(c)

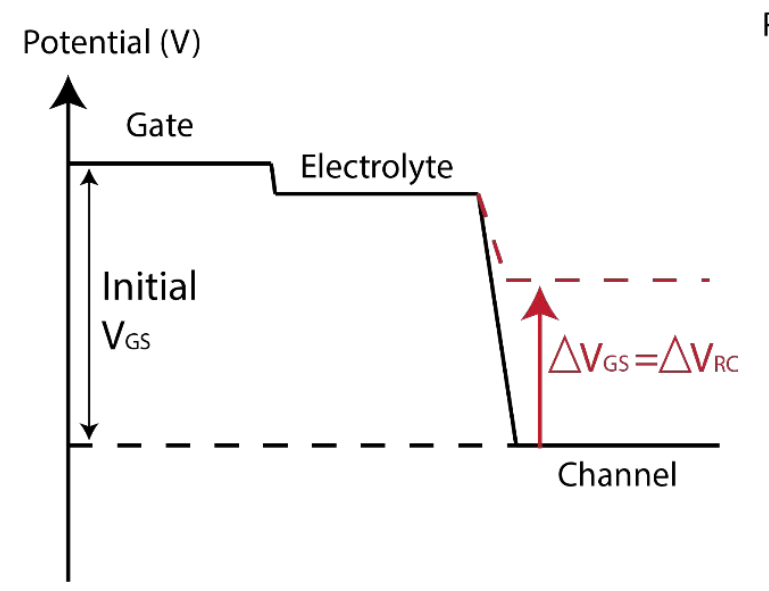

(b)

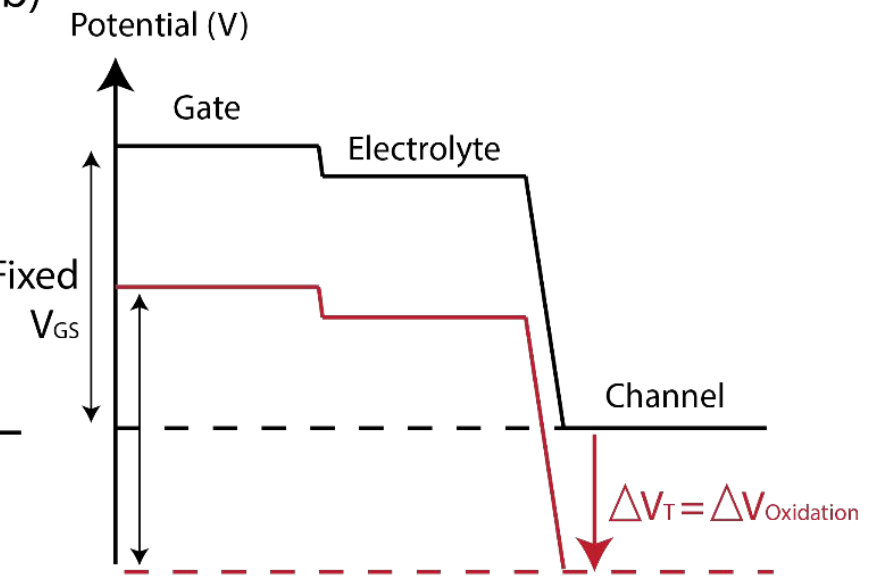

(d)

SI Figure 10. Illustration of potential profiles with the OECT from gate, to electrolyte and channel for different OECT chemical sensor architectures (a) Amperometric OECT with $\mathrm{Ag} / \mathrm{AgCl}$ gate. No potential changes are observed due to the fixed gate potential and gate voltage. (b) Amperometric OECT with OMIEC gate. Potentials throughout the OECT shift in concert due to oxidation of both gate and channel as well as the fixed gate voltage. (c) RC-OECT with $\mathrm{Ag} / \mathrm{AgCl}$ gate. Potential drop occurs mainly on the channel which changes by the same amount as $\Delta V_{\mathrm{RC}}$. (d) RC-OECT with OMIEC gate. Potential drop occurs mainly on the channel which changes by the same amount as $\Delta V_{\mathrm{RC}}$. There is additional shift in potentials across the OECT due to dirft in gate potential. 


\section{Modelling of Ideal Amperometric Sensors}

(a)

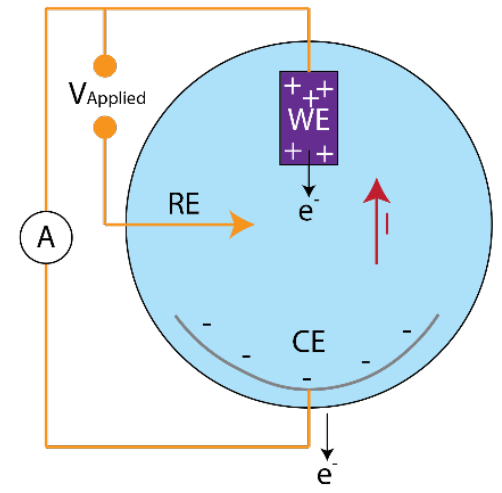

(b)

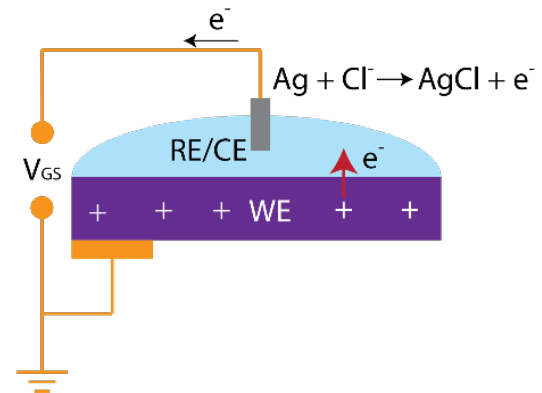

(c)

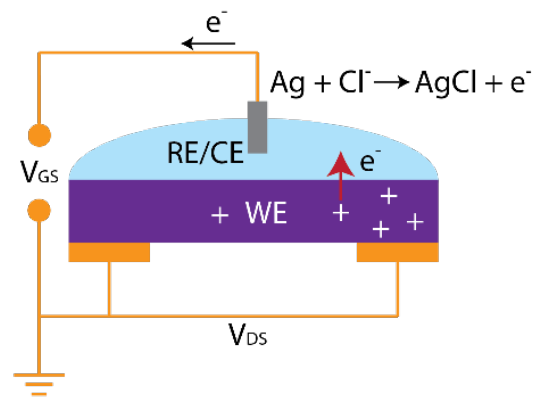

SI Figure 11. Comparison of 3-electrode and 2-electrode amperometric sensors. (a) 3electrode setup (b) 2-electrode setup with the absence a counter electrode (c) 2-electrode setup where conductivity of the working electrode is measured (OECT)

In a regular amperometric sensor (SI Figure 11 (a)), a potential is applied between a reference electrode (RE) and a working electrode (WE) while ensuring that there is minimal current flow between these two electrodes. For example, a saturated $\mathrm{Ag} / \mathrm{AgCl}$ reference consists of a saturated aqueous solution of $\mathrm{Cl}^{-}$ions (e.g. $\mathrm{KCl}$ ), $\mathrm{Ag}(\mathrm{s})$ and $\mathrm{AgCl}(\mathrm{s})$ such that the activities of the all three phases are constant, resulting in constant electrochemical potential of the reference electrode. Hence, the potential on the working electrode can be measured against a known value. In chronoamperometry measurements, the potential difference between the RE and WE is fixed by the potentiostat e.g. the initial open circuit potential. When $\mathrm{H}_{2} \mathrm{O}_{2}$ is introduced to the electrolyte, the oxidation of $\mathrm{p}(\mathrm{g} 3 \mathrm{~T} 2)$ leads to the injection of holes into the film. The accumulation of holes on p(g3T2) would lead to shifts in its potential which is not allowed in CA. Hence, an external current must flow from the potentiostat into the WE to maintain a constant OCV vs. the reference. For the circuit to be complete, an additional counter electrode (CE) is required to collect this current. In this 3-electrode setup, the current (or charge) injected into the WE is solely due to the compensation of charges generated by HPRR at the specific potential applied. The reaction current dependence on applied potentials can be expressed by the Butler-Volmer model. For a fixed potential, the Cottrell equation can be used to relate the reaction current to concentration.

To progress to the OECT sensor, let's first look at a pseudo amperometric sensor, which unlike the 3-electrode setup, lacks a counter electrode. In this case, an $\mathrm{Ag} / \mathrm{AgCl}$ pellet is used as a reference and a potential is applied between the pellet and the polymer. To catalyze the HPRR on the polymer, a positive potential difference is applied on the pellet with respect to the WE (e.g. $+0.3 \mathrm{~V}$ ), resulting in negative potentials on the WE. This leads to the reduction of hydrogen peroxide on the polymer and oxidation of $\mathrm{Ag}$ to $\mathrm{AgCl}$ on the pellet. Due to the absence of a counter electrode, current must flow through the reference electrode. Electrons from the oxidation of Ag flow from the pellet through the external circuit and replenish the holes generated on the polymer to maintain a constant potential difference between the pellet and polymer. The pellet here is not an ideal reference electrode as redox reactions of $\mathrm{Ag}$ are forced to occur which may lead to changes 
in $\mathrm{Cl}^{-}$concentrations in the electrolyte. In the limit where $\mathrm{Cl}^{-}$concentration is constant, the current measured would be the absolute amount of charge generated by HPRR at the applied potential (e.g. $-0.3 \mathrm{~V}$ vs. $\mathrm{Ag} / \mathrm{AgCl})$.

In an OECT sensor, the measured source-drain current does not capture the absolute amount of charge but how the injected charges affect conductivity of the channel which adds an additional layer of complexity to the understanding of device operation. The channel is a variable resistor where the applied $V_{\mathrm{GS}}$ modulates the channel conductivity and this conductivity can be probed by applying a $V_{\mathrm{DS}}$ across the channel and measuring drain current (ID). Concurrently, as discussed above, $V_{\mathrm{G}}$ also controls the rate of HPRR. Unlike other field effect transistors where $V_{\mathrm{GS}}>>V_{\mathrm{DS}}$, the fact that $V_{\mathrm{DS}}$ is on a similar magnitude as $V_{\mathrm{GS}}$ for an OECT results in further perturbation of

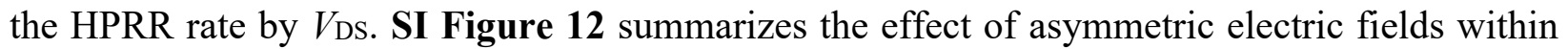
the OECT.

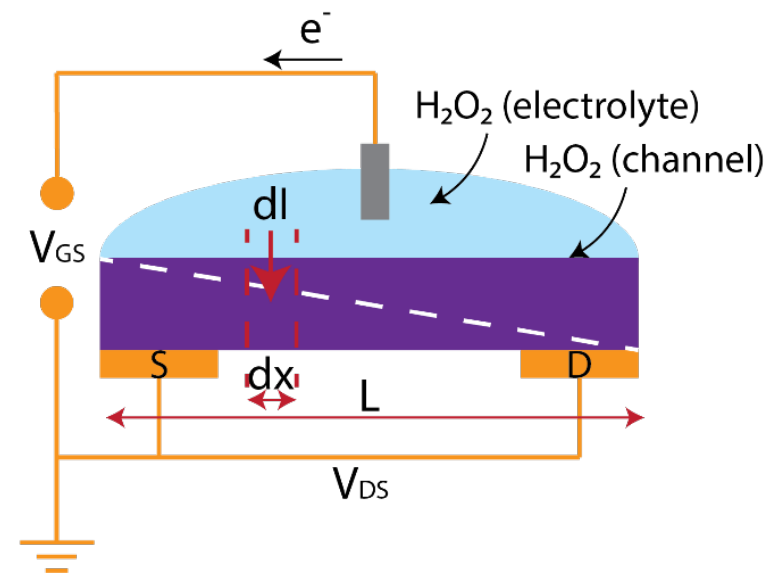

SI Figure 11. Illustration of approach to model faradaic reaction currents in an OECT. $d I$ is the current injected into a slice of $d x$ across the channel length.

Boundary conditions: Potentials are fixed by externally applied voltages to drive redox reactions. Hence, the OECT is at non-equilibrium conditions. To approximate the change in source-drain current in the OECT channel, we can use a modified Bulter-Volmer model:

The potential across the channel varies linearly, where $\mathrm{x}$ is the position from the source:

$$
V_{C H}(x)=\frac{x}{L} V_{D}+V_{G}
$$

For each infinitesimal slice $(\mathrm{dx})$ along the length of the channel, the infinitesimal reaction current (dI) dependent on the potential across the length of the channel can be expressed according to the Butler-Volmer model. Here, we only consider HPRR and can neglect the oxidation reaction of $\mathrm{H}_{2} \mathrm{O}_{2}$ as we are applying large positive gate potentials to drive HPRR on the OECT channel:

$$
d I(x)=I_{0}\left\{\frac{\left[H_{2} O_{2}\right]_{\text {channel }}(t)}{\left[H_{2} O_{2}\right]_{\text {electrolyte }}} \exp \left(-\frac{\alpha F \eta(x)}{R T}\right)\right\}
$$




$$
\eta(x)=\frac{x}{L} V_{D}+V_{G}-E_{0}
$$

Where $\mathrm{I}_{0}$ is the exchange current, $\alpha$ is the transfer coefficient, and $\eta$ is the overpotential (Eapplied $\mathrm{E}_{0}$ ). In this case, $\mathrm{E}_{\text {applied }}=\mathrm{V}_{\mathrm{CH}}$, the potential on the channel. The exchange current is dependent on the surface area of the channel. $\mathrm{k}^{0}$ is the standard rate constant of the reaction:

$$
\begin{gathered}
I_{0}=F W L k^{0}\left(\left[\mathrm{H}_{2} \mathrm{O}_{2}\right]_{\text {electrolyte }}\right)^{(1-\alpha)} \\
d I(x, t)=F W L k^{0}\left(\left[\mathrm{H}_{2} \mathrm{O}_{2}\right]_{\text {electrolyte }}\right)^{-\alpha}\left\{\left[\mathrm{H}_{2} \mathrm{O}_{2}\right]_{\text {channel }}(t) \cdot \exp \left(-\frac{\alpha F \eta(x)}{R T}\right)\right\} d x
\end{gathered}
$$

As the system is not in equilibrium, we need to account for the change in concentration of $\mathrm{H}_{2} \mathrm{O}_{2}$ at the channel surface which will be depleted over time and needs to be replenished by diffusion from the bulk of the electrolyte (modified from Cottrell equation, where we assume interfacial reaction kinetics are more rapid than mass transfer i.e. the channel is a sink for $\mathrm{H}_{2} \mathrm{O}_{2}$ ):

$$
\text { Flux } J_{\mathrm{H}_{2} \mathrm{O}_{2} \text { channel }}(0, t)=\frac{\left[\mathrm{H}_{2} \mathrm{O}_{2}\right]_{\text {electrolyte }} \sqrt{\mathrm{D}}}{\sqrt{\pi t}}
$$




\section{Charge accumulation (OMIEC gate): Current Transients}

We first investigate the phenomena in an amperometric OECT with OMIEC gate. Experimental data in Figure 3 and SI Figure 4 show the shift in threshold voltage after addition of $\mathrm{H}_{2} \mathrm{O}_{2}$ is indicative of charge accumulation on the channel. To model the time-dependent change in drain current, we assume:

1. Applied gate voltage is above the initial threshold voltage of the OECT, hence operating the OECT in the super-threshold regime. This is so that charge injected in the channel from the reaction results in the depletion of the valence band (accumulation of holes) and hence a change in charge density.

2. For simplicity, mobility is assumed to be independent of charge density. This may not hold true for all ranges of $V_{\mathrm{GS}}$ as $g_{\mathrm{m}}$ is not a constant with respect to $V_{\mathrm{G}}$. In particular, mobility is limited at potentials near the oxidation onset of the p-type channel $\left(V_{\mathrm{T}}\right)$.

3. No degradation of the polymer channel nor side reactions. This may not be the case at extreme potentials that result in permanent chemical changes in the polymer molecular structure and hence its electrochemical properties.

4. The bulk concentration of analyte in the electrolyte remains unperturbed.

5. All the charge injected $(\Delta \mathrm{Q})$ into the channel is due to reaction of the oxidant on the channel.

This leads to its change in conductivity (due to changes in density of charge carriers i.e. changing the volumetric capacitance of the channel). We then sum up the total amount of $\mathrm{H}_{2} \mathrm{O}_{2}$ entering the channel over the whole course of the experiment. The change in source-drain current (from Ohm's Law) would be:

$$
\Delta I_{C H}=V_{D} \cdot \frac{W d}{L} \cdot \mu \cdot \Delta Q_{\text {cumulative }}
$$

To get the instantaneous concentration of $\mathrm{H}_{2} \mathrm{O}_{2}$ at the surface of the channel, we integrate flux over time.

$$
\begin{gathered}
\text { Instantaneous }\left[\mathrm{H}_{2} \mathrm{O}_{2}\right]_{\text {channel }}(t)=\frac{2\left[\mathrm{H}_{2} \mathrm{O}_{2}\right]_{\text {electrolyte }} \sqrt{\mathrm{Dt}}}{\sqrt{\pi}} \\
\Delta Q_{\text {cumulative }}=\int_{0}^{t} \Delta Q_{\text {instantaneous }} d t=F W L k^{0}\left(\left[\mathrm{H}_{2} \mathrm{O}_{2}\right]_{\text {electrolyte }}\right)^{(1-\alpha)} \int_{0}^{t} \int_{0}^{L}\left\{\frac{2 \sqrt{D t}}{\sqrt{\pi}} \exp \left(-\frac{\alpha F\left(\frac{x}{L} V_{D}+V_{G}-E_{0}\right)}{R T}\right)\right\} d x d t
\end{gathered}
$$

Hence,

$$
\Delta I_{C H}(t)=V_{D} \cdot W^{2} d \cdot \mu \cdot F k^{0}\left(\left[H_{2} O_{2}\right]_{\text {electrolyte }}\right)^{(1-\alpha)} \int_{0}^{t} \int_{0}^{L}\left\{\frac{2 \sqrt{D t}}{\sqrt{\pi}} \cdot \exp \left(-\frac{\alpha F\left(\frac{x}{L} V_{D}+V_{G}-E_{0}\right)}{R T}\right)\right\} d x d t
$$

Which simplifies to:

$$
\Delta I_{C H}(t)=\boldsymbol{W}^{2} \boldsymbol{d} \boldsymbol{L} \cdot \mu \cdot F k^{0}\left(\left[H_{2} O_{2}\right]_{\text {electrolyte }}\right)^{(1-\alpha)} \cdot \frac{4 \sqrt{D} \boldsymbol{t}^{\mathbf{3} / 2}}{3 \sqrt{\pi}} \cdot\left\{\frac{1}{\beta} \exp \left[-\beta\left(\boldsymbol{V}_{\boldsymbol{G}}-E_{0}\right)\right] \cdot\left[1-\exp \left(-\frac{\beta \boldsymbol{V}_{\boldsymbol{D}}}{L}\right)\right]\right\}
$$


Where: $\beta=\frac{\alpha F}{R T}$

Let $\gamma=F k^{0}\left(\left[H_{2} O_{2}\right]_{\text {electrolyte }}\right)(1-\alpha) \frac{\sqrt{D}}{\sqrt{\pi}}\left\{\frac{1}{\beta} \exp \left[-\beta\left(\boldsymbol{V}_{\boldsymbol{G}}-E_{0}\right)\right] \cdot\left[1-\exp \left(-\frac{\beta \boldsymbol{V}_{\boldsymbol{D}}}{L}\right)\right]\right\}$

So

$$
\Delta I_{C H}(t)=W^{2} d L \cdot \mu \cdot \frac{4 t^{3 / 2}}{3} \cdot \gamma
$$

Total charge injected into the channel must equal the gate.

$$
\begin{gathered}
\Delta Q_{\text {cumulative, gate }}=\Delta Q_{\text {cumulative, } \text { channel }} \\
\Delta I_{\text {Gate }}=\frac{d\left(\Delta Q_{\text {cumulative }}\right)}{d t}=F W L^{2} k^{0}\left(\left[H_{2} O_{2}\right]_{\text {electrolyte }}\right)^{(1-\alpha)} \cdot \frac{2 \sqrt{D t}}{\sqrt{\pi}} \cdot \frac{1}{V_{D}}\left\{\frac{1}{\beta} \exp \left[-\beta\left(\boldsymbol{V}_{\boldsymbol{G}}-E_{0}\right)\right] \cdot\left[1-\exp \left(-\frac{\beta \boldsymbol{V}_{\boldsymbol{D}}}{L}\right)\right]\right\} \\
\Delta I_{\text {Gate }}=\frac{d\left(\Delta Q_{\text {cumulative }}\right)}{d t}=W L^{2} \cdot \frac{2 \sqrt{t}}{V_{D}} \cdot \gamma
\end{gathered}
$$

This analysis shows that the drain current of the amperometric OECT (with OMIEC gate) does not converge to a steady state on reasonable time scales under the above assumptions and boundary conditions. This is indeed observed in SI Figure 3 (d) where drain current increases steadily with a time dependence $\sim \mathrm{t}^{3 / 2}$ even when potential changes on the gate and channel begin to plateau off (SI Figure 3 (c)).

This model only holds for the above assumptions. When the OECT is operated at negative gate voltages (SI Figure 3 (a-b)), addition of an oxidizing agent results in over oxidation of the channel, resulting in a drop in conductivity of the channel. When the OECT is operated in the subthreshold regime as shown in Figure 3 (c), there is no charge accumulation on the channel, and the OECT behaves similar to the case with a non-polarizable gate (see next section). 


\section{Amperometric operation of OECT (Ag/AgCl gate)}

In the other limit, the charges generated by the reduction of $\mathrm{H}_{2} \mathrm{O}_{2}$ do not lead to accumulation of charges within the channel, so the charge density in the channel is only modified by the flux of $\mathrm{H}_{2} \mathrm{O}_{2}$. Whatever charges that enter the channel get swept into the current flowing through the conductor.

$$
\begin{gathered}
\Delta I_{C H}=V_{D} \cdot \frac{W d}{L} \cdot \mu \cdot \Delta Q_{F l u x} \\
\text { Let } \gamma=F k^{0}\left(\left[H_{2} O_{2}\right]_{\text {electrolyte }}\right)(1-\alpha) \frac{\sqrt{D}}{\sqrt{\pi}}\left\{\frac{1}{\beta} \exp \left[-\beta\left(\boldsymbol{V}_{\boldsymbol{G}}-E_{0}\right)\right] \cdot\left[1-\exp \left(-\frac{\beta \boldsymbol{V}_{\boldsymbol{D}}}{L}\right)\right]\right\} \\
\Delta I_{C H}=V_{D} \cdot \frac{W d}{L} \cdot \mu \cdot F W L k^{0}\left(\left[H_{2} O_{2}\right]_{\text {electrolyte }}\right)^{(1-\alpha)} \frac{2 \sqrt{D}}{\sqrt{\pi \boldsymbol{t}}} \int_{0}^{L}\left\{\exp \left(-\frac{\alpha F\left(\frac{x}{L} V_{D}+V_{G}-E_{0}\right)}{R T}\right)\right\} d x \\
\Delta I_{C H}(t)=\boldsymbol{W}^{2} \boldsymbol{d} \boldsymbol{L} \cdot \mu \cdot F k^{0}\left(\left[H_{2} O_{2}\right]_{\text {electrolyte }}\right)^{(1-\alpha)} \cdot \frac{2 \sqrt{D}}{\sqrt{\pi \boldsymbol{t}}} \cdot\left\{\frac{1}{\beta} \exp \left[-\beta\left(\boldsymbol{V}_{\boldsymbol{G}}-E_{0}\right)\right] \cdot\left[1-\exp \left(-\frac{\beta \boldsymbol{V}_{\boldsymbol{D}}}{L}\right)\right]\right\} \\
\Delta I_{C H}(t)=W^{2} \boldsymbol{d L} \cdot \mu \cdot 2 \boldsymbol{t}^{-1 / 2} \cdot \gamma \\
\Delta I_{\text {Gate }}=\Delta Q_{\text {instantaneous }}=F W L^{2} k^{0}\left(\left[H_{2} O_{2}\right]_{\text {electrolyte }}\right)^{(1-\alpha)} \cdot \frac{2 \sqrt{D \boldsymbol{t}}}{\sqrt{\pi}} \cdot \frac{1}{V_{D}}\left\{\frac{1}{\beta} \exp \left[-\beta\left(\boldsymbol{V}_{\boldsymbol{G}}-E_{0}\right)\right] \cdot\left[1-\exp \left(-\frac{\beta \boldsymbol{V}_{\boldsymbol{D}}}{L}\right)\right]\right\}
\end{gathered}
$$

Without permanent charge accumulation on the channel, the time-dependence of current in an amperometric OECT scales in a similar way to that of the Cottrell equation of an amperometric measurement conducted in a three-electrode electrochemical cell. 